\title{
On Stability And Some Remarks About Positional Systems
}

\author{
Marcelo Farias Caetano
}

TESE APRESENTADA

$\mathrm{AO}$

Instituto de Matemática e Estatística

DA

Universidade DE SÃo PaUlo

PARA

OBTENÇÃO DO TÍTULO

DE

Doutor em Matemática AplicAda

Program: Applied Mathematics

Advisor: Prof. Manuel Valentim de Pera Garcia

During this work the student was supported by CNPq fellowship with a period of one year of internship at Observatoire de Paris supported by CAPES, project number PVE089/2013

São Paulo, December 2017 


\section{On Stability And Some Remarks About Positional Systems}

Este exemplar corresponde à redação final da tese devidamente corrigida e defendida por Marcelo Farias Caetano

e aprovada pela Comissão Julgadora. Esta versão definitiva da tese contém as correções e alterações sugeridas pela Comissão Julgadora durante a defesa realizada por Marcelo Farias Caetano em 18/05/2018.

Comissão Julgadora:

- Prof. Dr. Manuel Valentim de Pera Garcia (orientador) - IME-USP

- Prof. Dr. Fábio Armando Tal - IME-USP

- Prof. Dr. Ricardo dos Santos Freire Jr. - IME-USP

- Prof. Dr. Diogo Cólon - EP-USP 
Aos meus pais 


\section{Aknowledge}

Agradeço aqui,

Ao meu orientador Manuel Valentim de Pera Garcia pela difícil tarefa de me orientar e ensinamentos durante todo o trabalho, ao amigo e coorientador Alain Albouy por todas as discussões construtivas sobre matemática, ensinamento e apoio durante a elaboração desse trabalho.

Aos meus pais, Fábio e Maria de Fátima, pelo apoio, suporte e amor em todos os momentos da minha vida.

À minha família, principalmente às minhas irmãs, avós e tia por me aguentarem e pelo apoio dado durante esses anos.

Aos Professores Clodoaldo, Fábio e Saulo pelo apoio dado durante todo o meu doutorado, seja da parte burocrática, quanto da parte matemática que foram cruciais para a confecção dessa tese.

Aos Professores Sônia Regina Leite Garcia, Ricardo Freire pelas dicas e correções dadas ao trabalho, e discussões calorosas durante os seminários dados por mim para a elaboração deste trabalho. Ao Jair Koiller por ter me ajudado em minha estadia na França por um período de estágio.

Aos amigos do IMEUSP Abdo, Danilo, Oda, Ricardo, Thiago, Bruna, Larissa e tantos outros com os quais eu pude contar com o apoio. Aos amigos antigos de IAG que sempre estiveram por perto quando precisei de apoio, principalmente Alessandra e Vinícius e não menos importante Fausto, Helen, Reinaldo.

Aos membros da banca, Manuel Valentim Pera Garcia, Fábio Tal, Ricardo Freire, Diogo Cólon e Renato Bortolatto, por terem aceito a realizar a banca em um período pouco convencional.

À Deus, por estar sempre presente.

À CNPQ, pelo apoio financeiro. 


\section{Resumo}

Nesta tese, estudamos problemas relacionados a campo de forças planares, isto é, sistema de equação diferencial ordinárias de segunda ordem

$$
\ddot{x}=f(x, y), \quad \ddot{y}=g(x, y), \quad f, g \in \mathcal{C}^{k} .
$$

Estudamos principalmente a estabilidade da origem no sentido de Liapunov. Chamaremos a atenção para a existência de funções auxiliares para alguns casos construídas a partir de integrais primeiras conhecidas. Estudaremos também um caso onde suponhamos que $f(x, y)=g(x, y)=f(x) \in \mathcal{C}^{\omega}$. Palavras-chave: Estabilidade de Liapunov, Movimentos Periódico, Integrais Primeiras. 


\section{Abstract}

In this thesis, we study problems concerns to planar force fields, that is, system of second degree ordinary differential equations

$$
\ddot{x}=f(x, y), \quad \ddot{y}=g(x, y), \quad f, g \in \mathcal{C}^{k} .
$$

Our focus is on the stability of equilibrium in the sense of Lyapunov. We give a special attention for the existence of auxiliary functions, obtained from first integral which is known. In the end we study a particular case when the force is analytic, i. e. $f(x, y)=g(x, y)=f(x) \in \mathcal{C}^{\omega}$.

Keywords: Lyapunov Stability, Periodic Motions, First Integrals. 


\section{Contents}

List of Figures $\quad$ xi

1 Introduction $\quad 1$

1.1 A Summary of the Results . . . . . . . . . . . . . . . . . . 2

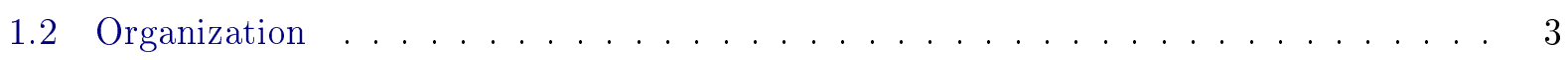

1.3 Notations . . . . . . . . . . . . . . . . . . . . . 4

2 Preliminaries and Basic Facts $\quad 5$

2.1 The Geometry of affine Space . . . . . . . . . . . . . . . . . . 5

2.2 Motion and Newtonian Mechanics . . . . . . . . . . . . . . . . . 9

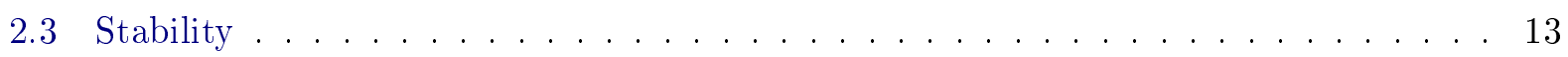

2.4 Remarks on Hill's Equation . . . . . . . . . . . . . . . . . . . . . . . 14

2.5 Elementary Properties of Real Analytic functions . . . . . . . . . . . . . 16

3 Planar Positional Force Fields $\quad 19$

3.1 Introduction . . . . . . . . . . . . . . . . . . . . . . 19

3.2 Point Transformations on Planar Positional Force . . . . . . . . . . . . . . . . 24

4 Stability of Some Positional Systems $\quad 29$

4.1 Systems With General Form . . . . . . . . . . . . . . . . . . 29

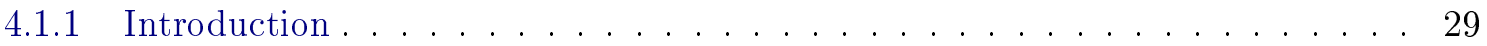

4.1.2 The Central Case $\mathrm{X}(\mathrm{x}, \mathrm{y})=-\mathrm{xf}(\mathrm{x}, \mathrm{y}), \mathrm{Y}(\mathrm{x}, \mathrm{y})=-\mathrm{yf}(\mathrm{x}, \mathrm{y}) \ldots \ldots \ldots \ldots \ldots$

4.1.3 Not a Beautiful World (or maybe yes) . . . . . . . . . . . . . 31

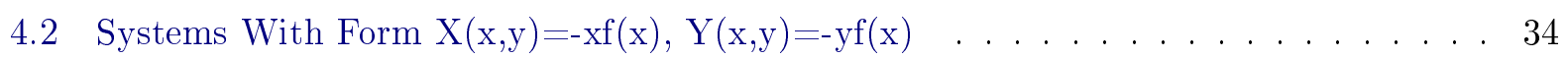

4.2.1 Liapunov Stability of Origin for System (4.10) . . . . . . . . . . . . 37

4.2.2 Some Remarks When $\mathrm{f}(\mathrm{x})$ is Analytic in System (4.10) . . . . . . . . . . . 38

$\begin{array}{ll}\text { Bibliography } & 43\end{array}$ 
x CONTENTS 


\section{List of Figures}

4.1 Configuration space for system (4.10) with $f(x)=0.5+x^{2}+x^{3} . \ldots \ldots \ldots$ 


\section{Chapter 1}

\section{Introduction}

The aim of this thesis is to study the problem of stability of equilibrium for some planar force field, which depends only the position of the particle attracted by a fixed point, namely Planar Positional Force Field.

In mathematical terms, we study the following system of ordinary differential equation of second order

$$
\ddot{q}=F(q), \quad q \in \mathbb{R}^{2} .
$$

For simplicity we call planar positional system (pps), or Newton system (pns), as the system of differential equations (1.1).

One can say that the systematic study of pps begun with Lagrange [26]. He studied the integrability of the two fixed center problem, or Euler problem (sse chapter). Another example was given by Halphen [17]. He gave an answer of a question of Bertrand year before [11].

Let $X(x, y)$ and $Y(x, y)$ be the components of the force imposed on the particle, the differential equations are

$$
\left\{\begin{array}{l}
\ddot{x}=X(x, y) \\
\ddot{y}=Y(x, y), \quad X, Y: \mathbb{R}^{2} \stackrel{\mathcal{C}^{k}}{\rightarrow} \mathbb{R}
\end{array}\right.
$$

Let us determine $X, Y$ such that the trajectory has the following form

$$
r=\left(x^{2}+y^{2}\right)^{1 / 2}=a x+b y+c,
$$

which represents all the possible conics for arbitrary constants $a, b, c$.

Differentiate equation (1.3) we obtain

$$
\frac{x \dot{x}+y \dot{y}}{r}=a \dot{x}+b \dot{y}
$$

and differentiate once more

$$
\frac{X x+\dot{x}^{2}+Y y+\dot{y}^{2}}{r}-\frac{(x \dot{x}+y \dot{y})^{2}}{r^{3}}=a X+b Y .
$$

Those two equations give us

$$
\left\{\begin{array}{l}
a=\frac{x}{r}+\frac{\dot{y}}{r^{3}} \frac{(x \dot{y}-y \dot{x})^{2}}{X \dot{y}-Y \dot{x}}, \\
b=\frac{y}{r}-\frac{\dot{x}}{r^{3}} \frac{(x \dot{y}-y \dot{x})^{2}}{X \dot{y}-Y \dot{x}} .
\end{array}\right.
$$

If we multiply $(1.6)_{1}$ by $x$ and $(1.6)_{2}$ by $y$, thus along a solution of (1.2), if we sum the two equations we obtain

$$
-c=\frac{(x \dot{y}-y \dot{x})^{3}}{r^{3}(X \dot{y}-Y \dot{x})},
$$


where $c$ is the arbitrary constant of (1.3). So the right side of (1.7) is constant if $X \dot{y}-Y \dot{x}$ is divisible by $x \dot{y}-y \dot{x}$ and $x \dot{y}-y \dot{x}$ is constant along a trajectory of (1.2). So, we obtain

$$
\left\{\begin{array}{l}
\ddot{x}=\mu x f(x, y) \\
\ddot{y}=\mu y f(x, y), \quad f: \mathbb{R}^{2} \stackrel{\mathcal{C}^{k}}{\rightarrow} \mathbb{R}, \quad f(x, y)=\frac{1}{r^{3}} .
\end{array}\right.
$$

In the begin of ninety's, Rauch-Wojciechowski [34] brought us, a new application for pps. They usually appear as a parametrization of stationary flows of KdV hierarchy. He showed that there is always exist a parametrization which turns each stationary KdV equation into a set of pns.

Let the fifth order Korteweg-de Vries (KdV) equation is the integrable PDE

$$
u_{t}=\frac{1}{16}\left(u_{x x x x}+10 u u_{x x}+5 u_{x}^{2}+10 u^{3}\right)_{x} .
$$

A Stationary flow of the KdV hierarchy is a time-independent solution $u(x)$, which gives $(2 m+$ $1)-s t, m=0,1,2, \ldots$ order nonlinear ordinary differential equation, which have a Lagrangian and a Hamiltonian structures. The ODE for the system above (1.9), is given by $u_{x x x x}+10 u u_{x x}+5 u_{x}^{2}+$ $10 u^{3}=16 k$, where $k$ is a constant of integration. This ODE is equivalent, under the parametrization $q_{1}=\frac{1}{4} u, q_{2}=\frac{1}{16} u_{x x}+\frac{5}{32} u_{x}^{2}$, to the system

$$
\left\{\begin{array}{l}
\ddot{q}_{1}=-10 q_{1}^{2}+4 q_{2} \\
\ddot{q}_{2}=10 q_{1}^{3}-20 q_{1} q_{2}+k .
\end{array}\right.
$$

This system is completely integrable, because it inherits two Hamiltonian formulations (one canonical and one non-canonical) from the KdV equation. Such a bi-Hamiltonian formulation is an effective formalism for producing Poisson commuting integrals of motion.

Our aim here is to study some properties of first integral for systems (1.1), which is very useful for Lyapunov stability of the origin. For this, we will give some assumptions on $F(q)$, that is, it is sufficiently smooth in a neighborhood of the origin $\left(\mathcal{C}^{k}(\mathcal{N}(0)), k \geq 2\right)$ to guarantee the existence and uniqueness of solution of (1.1). And, in $\mathcal{N}(0)$, the origin is the only equilibrium point of $F(q)$. In the literature, the system (1.1) can be found as Newton Systems (or Newton Equations), either as Non-conservative Positional Systems, or only Positional Systems. The name Nonconservative comes from the fact that we do not suppose that the force function $F(q)$ is derived from a potential function, that is, we don't need the existence of a function $\pi: \mathbb{R}^{2} \rightarrow \mathbb{R}, \mathcal{C}^{k}$, such that $\ddot{x}=\frac{\partial \pi}{\partial x}$ and $\ddot{y}=\frac{\partial \pi}{\partial y}$.

\subsection{A Summary of the Results}

Our results are concerned on stability of certain positional system (1.1).

The first result (proposition 16) is about a general system

$$
\left\{\begin{array}{l}
\ddot{x}=X(x, y) \\
\ddot{y}=Y(x, y), \quad X, Y: \mathbb{R}^{2} \stackrel{\mathcal{C}^{k}}{\rightarrow} \mathbb{R}
\end{array}\right.
$$

We suppose the force in (1.11) points inwards circumferences with center at origin which is an isolated equilibrium point. We call these forces quasi-attractor forces. We stated a criterium for the instability (in the sense of Lyapunov) of the origin for the system (1.11) which have a quadratic first integral, an energy-like first integral. For systems which have a first integral quadratic in velocities we show that those systems can be write in function of the coefficients of the first integral and the derivative of a function namely Pseudo-Potential.

Since we can rewrite the system in another form, we linearized it at the origin and obtain a criterium for instability according with the coefficients of the first integral. In fact, this is an 
Dirichlet-Lagrange like theorem for instability.

The second result (theorem 8) is about a system like

$$
\left\{\begin{array}{l}
\ddot{x}=-x(f(x, y)) \\
\ddot{y}=-y(g(x, y)), \quad f, g: \mathbb{R}^{2} \stackrel{\mathcal{C}^{k}}{\rightarrow} \mathbb{R},
\end{array}\right.
$$

which is a particular case of semi-attractor force, we call quasi-central attractor. In fact this kind of system can have two interesting consequence, the first one we will give an example which shows that the linearization at the origin does not give any information about the stability, but we show that the system is indeed unstable. This example can be generalized for the system (1.12) and we state a condition for instability of this system.

The other implication of this theorem comes as a surprise, since Barone and César [15] proved that the following system

$$
\left\{\begin{array}{l}
\ddot{x}=-x F(x) \\
\ddot{y}=-y G(x), \quad F, G: \mathbb{R} \stackrel{\mathcal{C}^{k}}{\rightarrow} \mathbb{R},
\end{array}\right.
$$

is generically stable. But we will give another example which shows that the genericity of the stability is not true if we have in (1.13) $F(x, y)$ and $G(x, y)$ instead. In fact it goes in the opposite way, the example shows that in the set of semi-attractor forces, the set of forces which give instability for the origin has non-empty interior.

The last result (proposition 24) given by us is about the system

$$
\left\{\begin{array}{l}
\ddot{x}=-x f(x) \\
\ddot{y}=-y f(x), \quad f: \mathbb{R} \stackrel{\mathcal{C}^{\omega}}{\rightarrow} \mathbb{R} .
\end{array}\right.
$$

Gaetano gave a necessary and sufficient condition [37] for the system (1.14) be stable, given $f(x)$ continuous. In fact for all this system be stable, $f(x)$ is generated by an involution. Here we put the assumption that $f(x)$ is real analytic, so through a auxiliary function $u(x)$ we proved that the knowing the even part of this auxiliary function, which is real analytic, we obtain all the information about the function $f(x)$.

This result is more a test to detect if a given system, $f(x) \in \mathcal{C}^{\omega}$ given, is stable or not.

\subsection{Organization}

In chapter two we review some basic concepts as real analytical function (see [25]), Hill's equation (see $[18,30]$ ), which we use in other chapters and we review a little of affine geometry (see the excellent book of Michel Berger [10]). Since there are few books which discuss about the Newtonian formalism in mechanics we intend in this chapter to do a Naive discussion on Newtonian mechanics as Galilean transformation and its difference for other point transformations, affine frame.

The subject of this thesis begin in chapter three. There, we define planar positional system and give two very important known results about this system. The first one was given by Paul Appell (see [3]), where he stated a transformation which takes a positional system and maps to another positional system. Indeed, it is the unique point transformation with a respective change in time which does it. He called this transformations as Homographies. This transformations is well known in Algebraic Geometry, in fact homographies are particular case of Collineations, which are transformations that maps Complex projective space into Complex projective space, that is, maps collinear points into collinear points. Homographies are the same of collineations but only in Real projective spaces.

The second result is due to Lagrange [26]. This result says a lot about quadratic first integrals of positional systems. In his work, Lagrange proposed to solve the problem of integrability of the Euler's three-body problem without any restriction on solution, since the answer given by Euler 
before demands some restriction on solutions. The second "mémoire" of this work perhaps is the first systematic study on first integral of planar positional systems, to my knowledge. There, he characterized quadratic first integral and the conditions on the systems to have this kind of constant of motion. For this, let us reminder, what is a first integral. Consider an ordinary differential equation

$$
G(t, x(t), \dot{x}(t))=0, \quad x: I \subset \mathbb{R} \rightarrow \mathbb{R}^{n}
$$

Definition. A first integral of the system (1.15) is a (non-constant) continuously-differentiable function $V: \mathbb{R} \times \mathbb{R}^{n} \rightarrow \mathbb{R}$, which is locally constant along any solution of (1.15), namely such that $\dot{V}=\frac{d V}{d t}(t, \varphi(t))=0$ for any $\varphi: J \rightarrow \mathbb{R}^{n}$ solution of (1.15), and $J$ the maximal interval for the solution. In general, the domain of definition of $V$ is much smaller than the domain of $G$, in particular we can choose a neighborhood of a particular point.

When we say quadratic, cubic,... first integrals we specify the degree in the velocities of $V$. That is, for a planar positional system, the definition above implies four first order autonomous ordinary differential equation, which we have $\tilde{V}=\tilde{V}(q, \dot{q})$ as a first integral. Thus, when we say that $\tilde{V}$ is quadratic, namely $\tilde{V}=r(\dot{q})+\pi(x, y)$, where $r(\dot{q})$ is a quadratic form with coefficients functions of position $q$ and $q=(x, y)$ and $\dot{q}=(\dot{x}, \dot{y})$.

The result by Lagrange state that a quadratic first integral indeed is a quadratic form in the variables $\dot{x}, \dot{y}, x \dot{y}-y \dot{x}$ with constant coefficients. This is an important accomplished since we use this to define a pseudo-conservative force field (see [8]), and quasi-potential systems (see [35]).

At last we have the stability chapter. There, we'll review the works of Barone, Cesar, Zampieri and state new results. We begin with the general case of (1.1) and give the example of a function which the stability of the origin is not detectable by the usual methods (quadratic first integral, linearization) by we can detect by a quartic first integral which we proof it is a Lyapunov function for the example. For the general case, we give results about system which has quadratic first integral but it does not come from any metric, that is, the quadratic part is indefinite. After this we study the central case, in fact the quasi-central case, which we will define in that section. We will give condition for some quasi-central system has the origin as a Lyapunov stable equilibrium point.

For the last, we arrive with a particular case of central system, which is known by Driven Systems (see [28]). For this, we review an important work by Zampieri [37] where he classifies continuous force fields which are Lyapunov stable. And we starting from this point to give a new results when we suppose that the force field is real analytical.

\subsection{Notations}

In this thesis, we suppose that the force function $F(q)$ in (1.1) is always smooth $\mathcal{C}^{k}(\mathcal{N}(0)), k \geq 2$ in a neighborhood of the origin, except in chapter 5 (5.2.2) which we give a new result about some real analytical planar system.

$q, \mathbf{x}, \dot{\mathbf{x}}$ always means vectors and $q_{i}, x_{i}, \dot{x}_{i}, \ldots$ means real values.

$\Delta$ means the determinant of some matrix and $\nabla$ means the gradient of some function defined in $\mathbb{R}^{n}$. 


\section{Chapter 2}

\section{Preliminaries and Basic Facts}

This chapter we will remember some basics results that we will use forward. We begin with affine geometry, which follows closely [10], where we introduce affine transformation and homography. In the following section we introduce the Newtonian mechanics, define motion and the principles which governing that mechanics some references for this topics are [5], [6], [33]. After, we reviews some results about Hill's equation and real analytic functions, which we will need to prove results in stability theory, some references are [18], [30], [25]

\subsection{The Geometry of affine Space}

In this section, we state the geometry of an affine space, which is the environment where our system is.

An affine space is nothing more than a vector space where origin we try to neglected by adding translations to the linear maps. It follows that the elementary properties of affine spaces, of their morphism and of their subspaces are all properties from linear algebra, more or less disguised. As a consequence, most demonstrations are automatic: To prove results about affine space, the idea is generally to use some appropriate translation to reduce the statement to a vector form. This often mean "vectorializing" the space at the appropriate point.

Definition. An affine space over a field $\mathbb{K}$ (which we supposed commutative) is a nonempty set $X$ endowed with faithful and transitive group action $(X, \vec{X}, \Phi)$, where $\vec{X}$ is a vector space on $\mathbb{K}$ considered with its additive group structure. This vector space is said to underlie the affine space $\boldsymbol{X}$, or the associated vector space for the affine space $\boldsymbol{X}$. And $\Phi(\xi, x)$ are translations of $\boldsymbol{X}$ from a fixed point $x$

$$
\Phi_{x}(\vec{\xi})=\Phi(\vec{\xi}, x)=x+\vec{\xi} .
$$

The left action, $\phi$, over $(X, \vec{X}, \Phi)$ is simply transitive, so there exists a map $\theta: X \times X \rightarrow \vec{X}$, such that $y=\Phi(\theta(x, y), x) \forall x, y \in X$. Let us define $\overrightarrow{x y}=\theta(x, y)=y-x$ and we say $\overrightarrow{x y}$ is a free vector or parallel displacement associated with the pair $(x, y)$. In particular, $\theta$ satisfies

$$
\left\{\begin{array}{l}
\theta_{x}: y \in X \mapsto \theta(x, y) \in \vec{X} \text { is a bijection } \forall x \in X \\
\theta(x, y)+\theta(y, z)=\theta(x, z)
\end{array}\right.
$$

since we have $\theta_{x}^{-1}(\vec{\xi})=x+\vec{\xi}$. Thus even the sum of two points of the affine space is not defined, the function $\theta$ says their difference is defined and the result is a vector.

Other definition we have is

Definition. Let $\boldsymbol{X}$ be a non-empty set, $\vec{X}$ a vector space on $\mathbb{K}$, and $\theta: X \times X \rightarrow \vec{X}$ a map satisfying the conditions above. Then $\boldsymbol{X}$ is affine space under the action

$$
\Phi(\vec{\xi})(x)=\theta_{x}^{-1}(\vec{\xi}) .
$$


The bijection $\theta_{x}$ also satisfy the following relations

$$
\begin{gathered}
\theta_{y}^{-1} \circ \theta_{x}=\Phi(\overrightarrow{x y}), \\
\theta_{y} \circ \theta_{x}^{-1}: z \mapsto z+\overrightarrow{x y}, \quad \forall x, y \in X .
\end{gathered}
$$

Definition. The dimension of $\boldsymbol{X}$, denoted by $\operatorname{dim}(X)=d$, is defined as the dimension of $\vec{X}$. If $d=0$ then $\boldsymbol{X}$ is a point, if $d=1$ then $\boldsymbol{X}$ is an affine line, and if $d=2$ then $\boldsymbol{X}$ is the affine plane.

Definition. If $a \in X$, we denote by $X_{a}$ the set $X$ endowed with the vector space structure $u \oplus_{a} w=$ $\phi\left(\theta_{a}(u)+\theta_{a}(v), a\right)$ (a the neutral element), such that $\theta_{a}: X \rightarrow \vec{X}$ is a vector isomorphism. We say that $X_{a}$ is the "vectorialization" of $\boldsymbol{X}$ at a.

Example 1. A trivial example is $X=\vec{X}$ with $\Phi(\vec{\xi})(\vec{\eta})=\vec{\xi}+\vec{\eta}, \quad \forall \vec{\xi}, \vec{\eta} \in \vec{X}$. We obtain a natural affine structure on any vector space.

In order to be able to calculate in affine spaces, we introduce something similar to vector space bases. We define affine frames for finite dimensional spaces.

Definition. An affine frame for the affine space $X$ consists of $d+1$ points $\left\{x_{i}\right\}_{i=0,1, \ldots, d}$ in $X$ such that $\left\{x_{0} x_{i}\right\}_{i=0,1, \ldots, d}$ is a basis for $\vec{X}$ (thus we necessarily have $d=$ dim $X$ ). The coordinates of $x \in X$ in this frame are the scalars $\left\{\lambda_{i}\right\}_{i=0,1, \ldots, d}$ such that $\overrightarrow{x_{0} x}=\sum_{i} \lambda_{i} x_{0} x_{i}$, i. e., the coordinates of $\overrightarrow{x_{0} x}$ in the basis $\left\{\overrightarrow{x_{0} x_{i}}\right\}_{i=0,1, \ldots, d}$ of $\vec{X}$. We can write $x=\left(\lambda_{1}, \ldots, \lambda_{d}\right)$.

Next, we'll define affine morphisms. This definition and proposition make the transition from a definition of a motion in an affine space to a vector space more natural,as we will see that some result on positional mechanical system remind close some results in affine geometry.

Let $(X, \vec{X}, \theta)$ and $(\tilde{X}, \overrightarrow{\tilde{X}}, \tilde{\theta})$ be two affine spaces over the same fields and $f: X \rightarrow \tilde{X}$ a map, in the set-theoretical sense.

Proposition 1. The following conditions are equivalent:

1. $f \in L\left(X_{a} ; \tilde{X}_{f(a)}\right)$ for some $a \in X$;

2. $f \in L\left(X_{a} ; \tilde{X}_{f(a)}\right)$ for all $a \in X$;

3. $\theta_{f(a)} \circ f \circ \theta_{a}^{-1} \in L\left(\vec{X}_{a} ; \overrightarrow{\tilde{X}}_{f(a)}\right)$ for some $a \in X$;

4. $\theta_{f(a)} \circ f \circ \theta_{a}^{-1} \in L\left(\vec{X}_{a} ; \overrightarrow{\tilde{X}}_{f(a)}\right)$ for all $a \in X$.

Moreover, if these conditions are satisfied, $\theta_{f(a)} \circ f \circ \theta_{a}^{-1} \in L\left(\vec{X}_{a} ; \overrightarrow{\tilde{X}}_{f(a)}\right)$ depends only on $f$, and will be denoted by $\vec{f}$ or $L(f)$. We have $\vec{f} \circ \theta=\theta(f, f)$. A map satisfyng the properties above is called an affine map between $X$ and $\tilde{X}$. And the set of this maps is denoted by $A(X, \tilde{X}) . f$ is an isomorphism if it is a bijective morphism; and a automorphism if it is an isomorphism from $X$ into itself.

If $f: X \rightarrow \tilde{X}$ is an affine map, we have, for all $x, y \in X$ :

$$
f(x) \vec{f}(y)=\vec{f}(\overrightarrow{x y}), \quad f(y)=f(x)+\vec{f}(\overrightarrow{x y}) .
$$

Example 2. Let $f \in A(X ; \tilde{X})$ and the two affine frames $\left\{x_{i}\right\}_{i=0,1, \ldots, n}$ and $\left\{\tilde{x}_{j}\right\}_{j=0,1, \ldots, m}$ respectively. Then the change of coordinate is given by

$$
\left(\begin{array}{c}
1 \\
\tilde{\lambda}_{1} \\
\vdots \\
\tilde{\lambda}_{m}
\end{array}\right)=M(f)\left(\begin{array}{c}
1 \\
\lambda_{1} \\
\vdots \\
\lambda_{m}
\end{array}\right), M(f)=\left(\begin{array}{cccc}
1 & 0 & \ldots & 0 \\
a_{1} & & & \\
\vdots & & M(\vec{f}) & \\
a_{m} & & &
\end{array}\right) \text {, }
$$


and

$$
M(\vec{f})=\left(\begin{array}{ccc}
\alpha_{11} & \ldots & \alpha_{1 n} \\
\vdots & \ddots & \vdots \\
\alpha_{m 1} & \ldots & \alpha_{m n}
\end{array}\right)
$$

All this definition to arrive in a theorem which says that an affine space is essentially determined by its lines and their set-theoretical intersection properties.

The functions $f \in A(X, \tilde{X})$ have the properties that images of every line $\ell$ of $X$ is a line (or possibly a point) of $\tilde{X}$, but the converse is false. For instance, when $X$ and $\tilde{X}$ are uni-dimensional, any bijection satisfies this property. Next, take $X=\tilde{X}=\mathbb{C}^{2}$ and $f\left(z_{1}, z_{2}\right)=\left(\bar{z}_{1}, \bar{z}_{2}\right)$, the function which maps two value of complex plane into their conjugate respectively. $f$ takes lines into lines but is not linear.

Definition. We call $\vec{f}: V \rightarrow \tilde{V}$ a semi-linear map, where $V$ and $\tilde{V}$ be vector spaces over $\mathbb{K}$ and $\tilde{\mathbb{K}}$, respectively, if there is an automorphism $\sigma: \mathbb{K} \rightarrow \tilde{\mathbb{K}}$ such that

$$
\vec{f}(\lambda x+\mu y)=\sigma(\lambda) f(x)+\sigma(\mu) f(y)
$$

for all $x, y \in V, \lambda, \mu \in \mathbb{K}$. Suppose $X, \tilde{X}$ are affine spaces over $\mathbb{K}$ and $\tilde{\mathbb{K}}$, respectively. Define the map between the vectorialization of $X, \tilde{X}$ at $x_{0}$ and $f\left(x_{0}\right)$ respectively, by $\vec{f}: X_{x_{0}} \rightarrow \tilde{X}_{f\left(x_{0}\right)}$. If exists $x_{0}$ such that $\vec{f}$ is semi-linear, then (by definition) $f: X \rightarrow \tilde{X}$ is called semi-affine

Theorem 1. Let $X, \tilde{X}$ be affine spaces of same finite dimension $d \geq 2$. Let $f: X \rightarrow \tilde{X}$ be a bijective map which takes any three collinear points $a, b, c \in X$ into collinear points $f(a), f(b), f(c) \in \tilde{X}$. Then $f$ is semi-affine.

Proposition 2. The only automorphism of $\mathbb{R}$ is the identity. The only continuous automorphisms of $\mathbb{C}$ are the identity and $z \mapsto \bar{z}$.

Corollary 1. Let $X, \tilde{X}$, be two real affine spaces of same finite dimension $d \geq 2$. If $f: X \rightarrow \tilde{X}$ is bijective and takes collinear points into collinear points, $f$ is an affine map.

Since we want to work with finite-dimensional real affine spaces (positional mechanical system on plane), we will endow our affine space with an Euclidean structure, so we want talk about the topology associated with the vector space, orientation and differentiability.

We recall that a real (or complex) vector space has a canonical topological structure. Consequently, the linear group $G L(E)$ of a $d$-dimensional vector space $E$ also has a canonical topology, induced by $L(E ; E)$, which is a vector space of dimension $d^{2}$.

Proposition 3. Let $X$ be an affine space, $a \in X$. The topology induced on $X$ by the canonical topology of the vectorialization $X_{a}$ does not depend on a, and is called the canonical topology on $X$.

Let $G A(X)=A(X ; X) \cap \mathcal{S}_{X}$, where $\mathcal{S}_{X}$ is the group of permutations on $X$ under composition of maps.

Proposition 4. The topology thus defined on $G A(X)$ depends only on $X$, and is called the canonical topology.

Now, we define an orientation for an affine space. First of all, we remind one definition for orientation of a vector space. Remark that the vector space $A^{d} E^{*}$ of alternating $d$-linear forms over a $d$-dimensional vector space $E$ is a real one-dimensional vector space; thus $A^{d} E^{*}$ has exactly two connected components.

Definition. An orientation for $E$ is the choice of one of the two components of $A^{d} E^{*} \backslash 0$. Let this component be $\mathcal{O}$. A form $\omega \in A^{d} E^{*}$ is called positive if $\omega \in \mathcal{O}$; a basis $\mathcal{B}=\left\{e_{i}\right\}_{i=1, \ldots, d}$ of $E$ is called positively oriented if $\omega\left(e_{1}, \ldots, e_{d}\right)>0$ for any $\omega \in \mathcal{O}$. 
Remark 1. If $\omega \in \mathcal{O}$ and $f \in G L(E)$, the pullback $f^{*} \omega$ is in $\mathcal{O}$ if and only if $\operatorname{det}(f)>0$. Thus, we introduce the notation: $G L^{+}(E)=\{f \in G L(E) \mid \operatorname{det}(f)>0\}$, and $G L^{-}(E)=\{f \in G L(E) \mid \operatorname{det}(f)<$ $0\}$.

This definition is equivalent to one says that two bases have the same orientation if they can be deformed (in the homotopic sense) into one another.

With this we have, for an arbitrary basis $\mathcal{O}$ of $E$ : If $\omega \in \mathcal{O}$ and $f \in G L^{+}(E)$, then $f^{*} \omega \in \mathcal{O}$. If $\mathcal{B}$ is a positively oriented basis for $E$, the basis $f(\mathcal{B})$ is positively oriented if and only if $f \in G L^{+}(E)$. For this reason we call elements of $G L^{+}(E)$ orientation-preserving, and will do so even if $E$ is not oriented. On the other hand, if $E, \tilde{E}$ are real vector spaces of same finite dimension, the following definition only makes sense if $E, \tilde{E}$ are oriented: $f \in I \operatorname{som}^{+}(E ; \tilde{E})$ if and only if $f^{*} \tilde{\omega} \in \mathcal{O}$ for any $\tilde{w} \in \tilde{\mathcal{O}}$, where $\mathcal{O}, \tilde{\mathcal{O}}$ are the orientations of $E, \tilde{E}$.

Definition. An orientation for an affine space $X$ is an orientation for the underlying vector space $\vec{X}$. We call $\left\{x_{i}\right\}_{i=0,1, \ldots, d}$ a positively oriented frame of the oriented affine space $X$ if $\left\{\vec{x}_{0} \vec{x}_{i}\right\}_{i=0,1, \ldots, d}$ is positively oriented basis for $\vec{X}$. We put

$$
\begin{aligned}
& G A^{+}(X)=\left\{f \in G A(X) \mid \vec{f} \in G L^{+}(\vec{X})\right\}, \\
& G A^{-}(X)=\left\{f \in G A(X) \mid \vec{f} \in G L^{-}(\vec{X})\right\} .
\end{aligned}
$$

The notion of differentiability can be extended from $\mathbb{R}^{n}$ to maps $f: U \rightarrow \tilde{X}$ from an open set $U$ of an affine space $X$ into another affine space $\tilde{X}$. One way to do this is to remark that different vectorializations of $X$ and $\tilde{X}$ differ only by translations, which are infinitely differentiable.

$$
f(x)-f(a)-g(x-a), \quad g \in L(X ; \tilde{X}),
$$

where $x-a=\overrightarrow{x a} \in \vec{X}$ and $f(x)-f(a)=f(a) \vec{f} f(x) \in \overrightarrow{\tilde{X}}$.

Remark 2. The derivative $f^{\prime} \in L(\vec{X}, \overrightarrow{\tilde{X}})$.

Basically we get the topological and differentiable structure on $X$ from $\vec{X}$ via pullback of $\theta_{a}$ for an arbitrary $a \in X$. Other useful technique is the existence of a universal vector space $\hat{X}$ where an affine space $X$ embeds as an affine hyperplane not containing the origin, which is applied in the study of affine quadrics within others techniques and constructions.

A metric is introduced into an affine space $X$ by choosing a scalar product (metric) in vector space $\vec{X}$ which is $X$ embeds. Different choices of $\vec{X}$ and scalar product give rise to different $d$-dimensional metric affine spaces. For instance, suppose we define the distance between two points $a=\left(x_{a}, y_{a}\right)$ and $b=\left(x_{b}, y_{b}\right)$ by

$$
\rho(a, b)=\left\{l\left(x_{a}-x_{b}\right)^{2}+2 m\left(x_{a}-x_{b}\right)\left(y_{a}-y_{b}\right)+n\left(y_{a}-y_{b}\right)^{2}\right\}^{1 / 2},
$$

which derive from the positive definite symmetric bilinear form called scalar product

$$
<u, M v>=\left(\begin{array}{ll}
x_{u} & y_{u}
\end{array}\right) M\left(\begin{array}{ll}
x_{v} & y_{v}
\end{array}\right)^{T},
$$

where ()$^{T}$ is the vector transpose and $M$ the matrix

$$
M=\left(\begin{array}{cc}
l & m \\
m & n
\end{array}\right), \quad l n-m^{2}>0, \quad l>0 .
$$

Fixing a triplet $(l, n, m)$ with $l n-m^{2}>0$, we pick a Euclidean structure for our affine space $X$, and we call it as Euclidean space.

Example 3. A Galilean space-time structure is a triple $\left(X^{4} ; \tau ;<,>\right)$ where $X^{4}$ is a dimension four affine space associated to vector space $\vec{X}^{4}, \tau$ is a non-zero linear form

$$
\tau: \vec{X}^{4} \rightarrow \mathbb{R},
$$


and $<,>$ is a scalar product defined on the three dimensional kernel $S=\tau^{-1}(0)$ of $\tau$. We call the elements in $X^{4}$ as the world points or events, $\tau$ the absolute time and $\tau(x-y)$ the time interval from events $x$ to $y$.

Let us define an equivalence relation on $X$, we say $x \sim y, x, y \in X$ if the vector $\overrightarrow{x y} \in S$ and we call two equivalent events as simultaneous events. The set $S_{x}=[x] \subset X$ of all events simultaneous to $x$ is a three dimensional Euclidean affine space associated to $S$.

One defines the Galilean group, $\operatorname{Gal}\left(X^{4}\right)$, of Galilean structure $\left(X^{4} ; \tau ;<,>\right)$ as a subgroup of $G A\left(X^{4}\right)$ whose elements preserve the time intervals of any pair of events and also preserve the distances between two simultaneous events, that is, the isometries of the space $X^{3}$ for any fixed $t \in X$. So if $T \in \operatorname{Gal}(X)$ we have

1. $\tau(x-y)=\tau(T(x)-T(y))$, for any $x, y \in X^{4}$;

2. $x_{1}, x_{2} \in X^{4}$ and $x_{1} \sim x_{2}$ imply $\left\|x_{1}-x_{2}\right\|=\left\|T\left(x_{1}\right)-T\left(x_{2}\right)\right\|$

So, uniform motion with velocity $v$, Translation of the origin $(0,0)$ to $(s, u) \in \mathbb{R} \times \mathbb{R}^{3}$ and rotation $\mathbf{R}$ of the coordinates axes belong to $G$ (we will see all those example again in the next chapter).

If we think the projective space induced by the affine space $X$, that is, if we add a "point" at infinity in the affine space, then we may think a linear transformation in this projective space $P(X)$

$$
L_{P(X)}: P(X) \rightarrow P(X)
$$

which is induced by the automorphism $f_{X}: X \rightarrow X$. We call this linear transformation Homography.

This may be written in terms of homogeneous coordinates using the matrix of homography, which is a matrix $(n+1) \times(n+1)$, where $n$ is the dimension of $X$. Up to the multiplication of nonzero constant in the field $\mathbb{K}$, we have

$$
\begin{gathered}
y_{0}=a_{00} x_{0}+a_{01} x_{1}+\ldots+a_{0 n} x_{n} \\
\vdots \\
y_{n}=a_{n 0} x_{0}+a_{n 1} x_{1}+\ldots+a_{n n} x_{n}
\end{gathered}
$$

where $\left[x_{0}: \ldots: x_{n}\right]$ are the homogeneous coordinates and $\left[y_{0}: \ldots: y_{n}\right]$ the images by $L$. In terms of affine coordinates we have

$$
y_{j}=\frac{a_{j 0}+a_{j 1} x_{1}+\ldots+a_{j n} x_{n}}{a_{00}+a_{01} x_{1}+\ldots+a_{0 n} x_{n}}
$$

which is defined outside the hyperplane $a_{00}+a_{01} x_{1}+\ldots+a_{0 n} x_{n}=0$.

Homography preserve collinear points. This is a direct fact from the fundamental theorem of projective space. Why this example is important? The answer for this question was given by Appell (), whom proved that given a positional force fields the only arbitrary point transformation accompanied with the change in time which leads to another positional force fields are the homographies. In fact, before Appell state his result, Halphen () has proved the same properties for central projection, which is a particular case of the homographies.

\subsection{Motion and Newtonian Mechanics}

Let us begin with our $d$-dimensional affine space $\mathbb{A}^{d}$ with fixed orientation, where the motion takes place. Fix the "origin" of reference, let say 0 . Now, we use the bijection $\theta_{0}$ to define our vector space $\overrightarrow{\mathbb{A}}^{d}$, then we endow that space with an Euclidean structure, that is a scalar product (2.4). Thus, the space where the motion takes place is a $d$-dimensional Euclidean space denoted by $\overrightarrow{\mathbb{E}}^{d}$. Then the position of every point $u \in \mathbb{A}^{d}$ is uniquely determined by its position vector $\overrightarrow{0 u}=\theta_{0}(u)=u-0=\mathbf{x} \in \overrightarrow{\mathbb{E}}^{d}$. We call $\mathbb{R}^{d}$ the set $\overrightarrow{\mathbb{E}}^{d}$, endowed with the scalar product $<,>$ (our 
Euclidean structure). From now on we call $\mathbb{E}^{d}$ as our affine space and $\mathbb{R}^{d}$ the vector space that underlies $\mathbb{E}^{d}$ with some metric.

The time is denoted by $t$ throughout and $t \in \mathbb{R}=\{t\}$.

A motion (or path) of the point $u$ is a smooth (at least $\mathcal{C}^{2}$ ) map $\mathbf{u}: I=(a, b) \rightarrow \mathbb{A}^{d}, a<$ $b, a, b \in \mathbb{R}$. Then every motion is uniquely determined by a smooth vector-function $\mathbf{x}: I \rightarrow \mathbb{R}^{d}$. The image of $I$ under the map $\mathbf{x}(t)$ is called the trajectory or orbit of the point $u$.

The velocity of the point $u$ at the time $t_{0} \in I$, we define as the derivative

$$
\mathbf{v}\left(t_{0}\right)=\left.\frac{d \mathbf{x}}{d t}\right|_{t=t_{0}}=\dot{\mathbf{x}}\left(t_{0}\right) \in \mathbb{R}^{d}
$$

so $\mathbf{v}\left(t_{0}\right)=\theta(u, s)=\overrightarrow{u s}$ is a vector in $\mathbb{R}^{d}$, where $s \in \mathbb{A}^{d}$, . And the vector $\dot{\mathbf{x}}\left(t_{0}\right)$ is independent of the choice of our origin.

We define the acceleration of the point $u$ at the instant $t_{0} \in I$ as

$$
\mathbf{a}=\left.\frac{d \mathbf{v}}{d t}\right|_{t=t_{0}}=\left.\frac{d^{2} \mathbf{x}}{d t^{2}}\right|_{t=t_{0}}=\ddot{\mathbf{x}}\left(t_{0}\right) \in \mathbb{R}^{d} .
$$

The set $\mathbb{E}^{d}$ is also called the configuration space of the points $u$. The point $(u, \mathbf{v})$ is called the state point and $\mathbb{E}_{\{u\}}^{d} \times \mathbb{R}_{\{\mathbf{v}\}}^{d}$ the phase (or state) space.

For the general case ( $n$ points masses) $u_{1}, \ldots, u_{n}$ each one moving in $\mathbb{E}^{d}$. A motion of the free system is given by smooth vector-function $\mathbf{r}(t)=\left(\mathbf{x}_{1}(t), \ldots, \mathbf{x}_{n}(t)\right) \in \mathbb{R}^{d n}$ and the velocity $\mathbf{v}=\dot{\mathbf{r}}=\left(\dot{\mathbf{x}}_{1}(t), \ldots, \dot{\mathbf{x}}_{n}(t)\right) \in \mathbb{R}^{d n}$ and the acceleration $\mathbf{a}=\ddot{\mathbf{r}}=\left(\ddot{\mathbf{x}}_{1}(t), \ldots, \ddot{\mathbf{x}}_{n}(t)\right) \in \mathbb{R}^{d n}$

The Newton-Laplace principle of determinacy (which one can obtain experimentally) asserts that the states of the system at any fixed time uniquely determines all of its motion in future.

Suppose that we know the state of the system at an instant $t_{0},\left(\mathbf{r}_{0}, \mathbf{v}_{0}\right)$, then, according to the principle above, we know the motion $\mathbf{r}(t), t \in I \subset \mathbb{R} ; \mathbf{r}_{0}=\mathbf{r}\left(t_{0}\right)$ and $\mathbf{v}_{0}=\dot{\mathbf{r}}_{0}=\dot{\mathbf{r}}\left(t_{0}\right)$. In particular, we can calculated the acceleration $\ddot{\mathbf{r}}$ at $t=t_{0}$. Then $\ddot{\mathbf{r}}\left(t_{0}\right)=\tilde{\mathbf{F}}\left(t_{0}, \mathbf{r}\left(t_{0}\right), \dot{\mathbf{r}}\left(t_{0}\right)\right)$, where $\tilde{\mathbf{F}}$ is some function where the existence follows from the Newton-Laplace principle. Since we can choose the time arbitrarily, we have

$$
\frac{d \mathbf{p}}{d t}=\mathbf{F}(\mathbf{x}, \mathbf{v}, t), \forall t
$$

where $\mathbf{p}=\mathbf{M v}$ is the momentum, and

$$
\mathbf{M}=\operatorname{diag}\left(m_{1} I d_{d \times d}, \ldots, m_{n} I d_{d \times d}\right),
$$

$I d_{d \times d}$ is the identity matrix $d$-dimensional. If $\mathbf{M}$ is constant then $\frac{d \mathbf{p}}{d t}=\mathbf{M} \ddot{\mathbf{r}}$ and $\tilde{\mathbf{F}}=\mathbf{M}^{-1} \mathbf{F}$.

This differential equation is called the equation of motion or Newton's equation. The existence of Newton's equation (with a smooth vector-function $\mathbf{F}: \mathbb{R}_{\{\mathbf{r}\}}^{d n} \times \mathbb{R}_{\{\mathbf{v}\}}^{d n} \times \mathbb{R}_{\{t\}} \rightarrow \mathbb{R}^{d n}$ is equivalent to the principle of determinacy. This follows from the existence and uniqueness theorem in theory of differential equations. The function $\mathbf{F}$ is usually determined experimentally.

Let us consider $\mathbb{R}^{3} \times \mathbb{R}$, as our affine space, $\tau: \mathbb{R}^{3} \times \mathbb{R} \rightarrow \mathbb{R}$ be the projection $\tau(x, t)=t$, for all $(x, t) \in \mathbb{R}^{3} \times \mathbb{R}$ and $S=\left\{(0, x) \mid x \in \mathbb{R}^{3}\right\}$ endowed with the scalar product on $\mathbb{R}^{3}$. The Galilean space-time structure is the so called Galilean coordinate space and is denoted by $G\left(\mathbb{R}^{3} \times \mathbb{R}\right)=G$

The following transformations belong to $\operatorname{Gal}\left(\mathbb{R}^{3} \times \mathbb{R}\right)$

\section{- Uniform motion with velocity $v$ :}

$$
g_{1}(x, t)=(x+t v, t),(x, t) \in \mathbb{R}^{3} \times \mathbb{R} ;
$$

- Translation in the space and time:

$$
g_{2}(x, t)=(x+w, t+s),(x, t) \in \mathbb{R}^{3} \times \mathbb{R}
$$


- Rotation $R$ of the coordinates axis $v$ :

$$
g_{3}(x, t)=(R x, t),(x, t) \in \mathbb{R}^{3} \times \mathbb{R},
$$

where $R$ is an orthogonal (proper or not) transformation of $\mathbb{R}^{3}$.

Proposition 5. Every Galilean transformation $g: \mathbb{R}^{3} \times \mathbb{R} \rightarrow \mathbb{R}^{3} \times \mathbb{R}$ can be uniquely represented as a composition $g_{1} \circ g_{2} \circ g_{3}$.

Indeed, we have to proof that all those example above are in $\operatorname{Gal}\left(\mathbb{R}^{3} \times \mathbb{R}\right)$. Let us consider a general affine transformation of $G A\left(\mathbb{R}^{4}\right)=G A\left(\mathbb{R}^{3} \times \mathbb{R}\right)$ then we shall find an explicit form of Galilean transformation.

Let us revisit the example (2), and define the change of coordinate. Let $X^{d}$ be an affine space with dimension $d$, an affine coordinate system with origin $0 \in X^{d}$ can be defined as follow

Definition. Let $\left\{e_{1}, \ldots, e_{d}\right\}$ be a basis of the vector space $\vec{X}^{d}$ associated to $X^{d}$. For any point $p \in X^{d}$ we write

$$
\theta(0, p)=\overrightarrow{0 p}=\sum x_{i}(p) e_{i}
$$

where $\left\{x_{i}(p)\right\}_{1 \leq i \leq d}$ is an uniquely determined $d$-tuple of real numbers, called the coordinates of $p$. The set of functions $\left\{x_{i}(p)\right\}_{1 \leq i \leq d}$ is called the affine frame or affine coordinate system.

If we have two affine frames $\left\{x_{i}(p)\right\}_{1 \leq i \leq d}$ and $\left\{y_{i}(p)\right\}_{1 \leq i \leq d}$ then they are related by

$$
y_{i}=\sum_{k} a_{i k} x_{k}+c_{i}, \quad 1 \leq i \leq d,
$$

where $A=\left[a_{i k}\right]$ is a nonsingular $d \times d$ matrix and $c=\left[c_{i}\right]_{1 \leq i \leq d}$ is a vector.

This may be expressed by the equation $y=A x+c$, or

$$
\left(\begin{array}{l}
1 \\
y
\end{array}\right)=\left(\begin{array}{ll}
1 & 0 \\
c & A
\end{array}\right)\left(\begin{array}{l}
1 \\
x
\end{array}\right)
$$

Let $f: X^{d} \rightarrow X^{d}$ be an affine transformation. Let $\left\{x_{i}\right\}_{1 \leq i \leq d}$ be an affine frame with origin in 0 and based on $\left\{e_{i}\right\}_{1 \leq i \leq d}$. Let $\left\{y_{i}\right\}_{1 \leq i \leq d}$ be an affine coordinate system with origin $f(0)$ and based on $\left\{\vec{f}\left(e_{i}\right)\right\}_{1 \leq i \leq d}$. Then we have $y_{i}(f(p))=x_{i}(p)$, for $p \in X^{d}$. We can write the relationship between the coordinate system $\left\{x_{i}\right\}_{1 \leq i \leq d}$ and $\left\{y_{i}\right\}_{1 \leq i \leq d}$ in the form

$$
x_{i}=\sum_{k} b_{i k} y_{k}+l_{i}, \quad 1 \leq i \leq d .
$$

Therefore, relative to the one coordinate system $\left\{x_{i}\right\}_{1 \leq i \leq d}$, the coordinate $\bar{x}_{i}=x_{i}(f(p))$ of the image $f(p)$ can be expressed in terms of the coordinates of $p$ in the form

$$
\bar{x}_{i}=x_{i}(f(p))=\sum b_{i k} y_{k}(f(p))+l_{i}=\sum b_{i k} x_{k}(p)+l_{i}, \quad 1 \leq i \leq d .
$$

Again this equation can be put in matrix form $\bar{x}=B x+l$ or

$$
\left(\begin{array}{l}
1 \\
\bar{x}
\end{array}\right)=\left(\begin{array}{ll}
1 & 0 \\
l & B
\end{array}\right)\left(\begin{array}{l}
1 \\
x
\end{array}\right)
$$

Let us prove that the transformations $g_{1}, g_{2}, g_{3}$ above are in $\operatorname{Gal}\left(\mathbb{R}^{3} \times \mathbb{R}\right.$. A general affine transformation has the following form

$$
\mathbf{x}=R \mathbf{x}^{\prime}+\mathbf{v} \tau^{\prime}+\mathbf{a},
$$

where $\mathbf{v}$, a are vectors in $\mathbb{R}^{3}$ and $R \in \mathcal{A}_{3 \times 3}$, a matrix. The time measure in the frame $\mathbf{x}^{\prime}$ is $\tau^{\prime}$, a linear form (see chapter 1 ) defined as a projection $\tau^{\prime}\left(\mathrm{x}^{\prime}, t^{\prime}\right)=t^{\prime}$. 
The transformation of time, for the new frame has the following form

$$
\tau=\left((k, l) ;\left(\tau^{\prime}, \mathbf{x}^{\prime}\right)\right)+s=\left(l ; \mathbf{x}^{\prime}\right)+k \tau^{\prime}+s,
$$

where $l \in \mathbb{R}^{3}$ and $k, s \in \mathbb{R}$ are constants. The product (;) is a matrix product, do not confuse with the scalar product.

Remark 3. The equations (2.9) has the same form of (2.7)

$$
\left(\begin{array}{c}
1 \\
x_{1} \\
x_{2} \\
x_{3} \\
\tau
\end{array}\right)=\left(\begin{array}{ccccc}
1 & 0 & 0 & 0 & 0 \\
\alpha_{1} & & & & v_{1} \\
\alpha_{2} & & R_{3 \times 3} & & v_{2} \\
\alpha_{3} & & & & v_{3} \\
\alpha_{4} & & l & & k
\end{array}\right)\left(\begin{array}{c}
1 \\
x_{1}^{\prime} \\
x_{2}^{\prime} \\
x_{3}^{\prime} \\
\tau^{\prime}
\end{array}\right)
$$

where $\left(\alpha_{1}, \alpha_{2}, \alpha_{3}, \alpha_{4}\right)=\boldsymbol{a}$ in (2.9).

For this to be in the Galilean group, it must have the properties (see chapter 1). Let $\mathbf{x}^{(i)}=$ $\left(x_{1}^{(i)}, x_{2}^{(i)}, x_{3}^{(i)}, \tau^{(i)}\right)$ and $\mathbf{x}^{\prime(i)}=\left({x^{\prime}}_{1}^{(i)}, x_{2}^{\prime(i)}, x_{3}^{\prime(i)}, \tau^{\prime(i)}\right)$.

1. The time difference between two events in the new frame must be equal to the old frame:

$$
\begin{gathered}
\tau=\tau^{\prime} \\
\tau\left(\mathbf{x}^{(1)}\right)-\tau\left(\mathbf{x}^{(2)}\right)=\tau\left(\mathbf{x}^{(1)}-\mathbf{x}^{(2)}\right)=\left[\left((l, k) ; \mathbf{x}^{(1)}\right)+s\right]-\left[\left((l, k) ; \mathbf{x}^{,(2)}\right)+s\right],
\end{gathered}
$$

where the last equality comes from the fact that $\tau$ is a projection.

Then we have

$$
\tau_{1}-\tau_{2}=\tau_{1}^{\prime}-\tau_{2}^{\prime}
$$

for every two events if, and only if $l=\overrightarrow{0}$. After, this must be true for any two events then we obtain $k=1$, since the first term (2.10) vanish. Thus the translation in time is an Galilean transformation.

2. Two equivalent, simultaneous, events must remain simultaneous:

$$
\left\|\mathrm{x}_{1}-\mathrm{x}_{2}\right\|=\left\|\mathrm{x}_{1}{ }_{1}-\mathrm{x}_{2}{ }_{2}\right\|
$$

We obtain $R \in O(3)$ (the orthogonal group of $\mathbb{R}^{3}$ ) since

$$
\left\|\mathbf{x}_{1}-\mathbf{x}_{2}\right\|=\left\|R\left(\mathbf{x}_{1}{ }_{1}-\mathbf{x}^{\prime}{ }_{2}\right)\right\|
$$

by (2.9). And

$$
\left\|R\left(\mathbf{x}_{1}-\mathbf{x}_{2}\right)\right\|=\left\|\mathbf{x}_{1}{ }_{1}-\mathbf{x}_{2}{ }_{2}\right\|
$$

in the usual scalar product, if, and only if $R \in O(3)$.

Then the general form of the Galilean transformations are:

$$
\mathbf{x}=R \mathbf{x}^{\prime}+\mathbf{v} \tau^{\prime}\left(\mathbf{x}^{\prime}\right)+\mathbf{a}, \quad \tau(\mathbf{x})=\tau^{\prime}\left(\mathbf{x}^{\prime}\right)+s ; \quad R \in O(3), \quad \mathbf{v}, \mathbf{a} \in \mathbb{R}^{3}, \quad s \in \mathbb{R} .
$$

Every element of the Galilean group transforms the frames into another frame, which moves rectilinear and uniformly with respect to the first frame. Such frames are said to be inertial. The Galileo-Newton principle of relativity asserts that Newton's equations are invariant under the Galilean transformation group in an inertial frame of reference. 


\subsection{Stability}

The concept of stability seems to be clear and of good use in everyday life. Very early, the stability concept was specialized in mechanics to describe some type of equilibrium of a material particle or system. Consider for instance a particle subject to some forces and possessing an equilibrium point $q_{0}$. The equilibrium is called stable if, after any sufficiently small perturbations of its position and velocity, the particle remains arbitrarily near $q_{0}$, with arbitrarily small velocity. Formulated in precise mathematical terms, this mechanical definition of stability was found useful in many situations, but inadequate in many others. This is why a lot of other concepts have been introduced. Though other concepts are known, the principal question remains the same: "What happen with the motion of the system starting near the equilibrium point"?

Liapunov in his work "Stability of Motion" was one of the first to introduced a formal concepts about stability. As contrasted with mechanical stability, the "Liapunov's stability" concerns no more to a material particle (or the equations thereof), but to a general differential equation; and now it applies to a solution not only to an equilibrium point or critical point.

Let

$$
\dot{q}=f(t, q),
$$

where $q$ and $f$ are real $n$-vectors, $t$ is the time, $f$ is defined on $\mathbb{R} \times \mathbb{R}^{n}$ and $\dot{q}=\frac{d q}{d t}$. We assume $f$ smooth enough to ensure existence, uniqueness and continuous dependence of the solutions of the initial value problem associated with (2.12) over $\mathbb{R} \times \mathbb{R}^{n}$.

A solution $\varphi(t)$ of (2.12) is called stable at $t_{0}$, or, more precisely, stable at $t=t_{0}$ in the sense of A. M. Liapunov [29] if, for every $\epsilon>0$, there is a $\delta>0$ such that if $\psi(t)$ is any other solution with $\left\|\psi\left(t_{0}\right)-\varphi\left(t_{0}\right)\right\|<\delta$, then $\|\psi(t)-\varphi(t)\|<\epsilon$ for all $t \geq t_{0}$. Otherwise we say that $\varphi(t)$ is unstable at $t=t_{0}$.

We presented above the concept of stable solution $\varphi(t)$ for equation (2.12). If we replace $q$ by a new variable $z=q-\varphi(t)$, then (2.12) becomes

$$
\dot{z}=g(t, z)=f(t, z+\varphi(t))-f(t, \varphi(t)),
$$

where $g(t, 0)=0$. Then the origin is a critical point of (2.13) and stability of the solution $\varphi(t)$ of (2.12) is equivalent to stability of the critical point for (2.13). In general, this passing is not always possible, but we restrict ourselves to concentrate on stability of critical points, with main idea to establishing some criteria to decide where the critical point is or is not a stable equilibrium, from the physical data, without a possible integration of the motion.

What is known as Liapunov's direct or second method for the study of stability, makes an essential use of auxiliary functions, also called Liapunov or Liapunov-like function. Those of the simplest type are $\mathcal{C}^{1}$ functions

$$
V: I \times \Omega \rightarrow \mathbb{R}
$$

where $I$ and $\Omega$ are the maximal interval of definition of a solution $\varphi$ and $\Omega$ contains the image of $\varphi$.

If $\varphi(t)$ is some solution of (2.12), the derivative of $V$ with respect time $t$ along $\varphi(t)$ is given by

$$
\frac{d}{d t} V=<\nabla V, f(t, q)>+\frac{\partial V}{\partial t} .
$$

Consider now a function $V(t, q)$ defined as above, but such that $V(t, 0)=0$, and, for some function $l: \mathbb{R}^{+} \rightarrow \mathbb{R}^{+}$and every $(t, q) \in I \times \Omega, V(t, q) \geq l(\|q\|)$. Such a function is called to be positive definite on $\Omega$.

One of results obtained by Liapunov is:

Theorem 2. If there is a $\mathcal{C}^{1}$ function $V: I \times \Omega \rightarrow \mathbb{R}$, such that, for some $l: \mathbb{R}^{+} \rightarrow \mathbb{R}^{+}$and every $(t, q) \in I \times \Omega$ :

1. $V(t, q) \geq l(\|q\|) ; V(t, 0)=0$. 
2. $\dot{V}(t, q) \leq 0$;

then, the origin is stable.

Since we are interested in autonomous system, the following statement of the theorem is more appropriate

Theorem 3. Let an open set $\Omega \subset \mathbb{R}^{n}, f: \Omega \rightarrow \mathbb{R}^{n}$ smooth, and $q_{0}$ an equilibrium point of $\dot{q}=f(q)$. Suppose that exist $U \subset \Omega$, open set, with $q_{0} \in U$, and $V: U \rightarrow \mathbb{R}$ smooth such that,

1. $q_{0}$ is a strict minimum point of $V$, that is, $V\left(q_{0}\right)<V(q), \forall q \in U, q \neq q_{0}$;

2. $\dot{V}(q)=\nabla V(q) f(q) \leq 0, \forall q \in U$.

Then $q_{0}$ is a Liapunov stable equilibrium point of $\dot{q}=f(q)$.

\subsection{Remarks on Hill's Equation}

Any homogeneous linear differential equation of second order with real periodic coefficients can be reduced to an equation of Hill's type. One question wich arises in this type of equation is the existence of periodic solutions. Furthermore, the value of the period of the solution (which is a multiple of the period $T$ of the coefficients) plays an important role in the discussion of periodic solutions.

The name of Hill's equation is given to the equation

$$
\frac{d}{d t}\{P(t) \dot{y}(t)\}+Q(t) y(t)=0,
$$

where $P(t)$ and $Q(t)$ are real-valued and have the same period $T$. In addition, it is assumed that $P(t)$ is continuous and nowhere zero, and that $\dot{P}(t)$ and $Q(t)$ are piecewise continuous. Thus (2.14) is a particular case of

$$
a_{0}(t) \ddot{y}(t)+a_{1}(t) \dot{y}(t)+a_{2}(t) y(t)=0,
$$

where the coeffcients $a_{k}(t)$ are complex-valued, piecewise-continuous, and periodic, with the same period $T>0\left(a_{k}(t+T)=a_{k}(t)\right)$.

To reduce the form (2.15) to the form (2.14), let $a_{1}(t) / a_{0}(t)$ has a piecewise continuous derivative and make the substitutuion

$$
y(t)=z(t) \exp \left(-\frac{1}{2} \int_{0}^{t}\left\{a_{1}(\eta) / a_{0}(\eta)\right\} d \eta\right)
$$

into (2.15). Then we arrive

$$
\ddot{z}(t)+\left\{a_{2}(t)-\frac{1}{4}\left(\frac{a_{1}(t)}{a_{0}(t)}\right)^{2}-\frac{1}{2} \frac{d}{d t}\left(\frac{a_{1}(t)}{a_{0}(t)}\right)\right\} z(t)=0,
$$

which has the form $(2.14)$, since $z(t)$ is periodic.

Equation (2.15) has the property that there are a non-zero constant $\rho$ and a non-trivial solution $\psi(t)$ such that

$$
\psi(t+T)=\rho \psi(t) .
$$

This property leads to the existence of two linearly independent solutions of (2.15)

$$
\psi_{1}(t)=e^{m_{1} t} p_{1}(t), \quad \psi_{2}(t)=e^{m_{2} t} p_{2}(t),
$$

where $m_{1}$ and $m_{2}$ are constants, not necessarily distinct, and $p_{1}(t)$ and $p_{2}(t)$ are periodic with period $T$, or

$$
\psi_{1}(t)=e^{m t} p_{1}(t), \quad \psi_{2}(t)=e^{m t}\left[p_{2}(t)+t p_{1}(t)\right],
$$


where $m$ is a constant, and $p_{1}(t)$ and $p_{2}(t)$ are periodic with period $T$.

The equation (2.15) with properties given above has two continuosly differentiable solutions $y_{1}(t)$ and $y_{2}(t)$ wich are uniquely determined by the conditions:

$$
y_{1}(0)=1, \dot{y}_{1}(0)=0, y_{2}(0)=0, \dot{y}_{2}(0)=1 .
$$

These solutions are referred to as normalized solutions of (2.15).

Let $y_{1}(t)$ and $y_{2}(t)$ the normalized solutions of (2.14) then the characteristic multipliers $\rho_{1}$ and $\rho_{2}$ are the roots of

$$
\rho^{2}-\left[y_{1}(T)+\dot{y}_{2}(T)\right] \rho+1=0,
$$

as one can prove from the Wronskian

$$
y_{1}(t) \dot{y}_{2}(t)-y_{2}(t) \dot{y}_{1}(t)=1, \forall t,
$$

and Liouville's formula, and satisfy

$$
\rho_{1} \rho_{2}=1 .
$$

As a consequence of characteristic polynomial we have

Theorem 4. (Stability test). The solutions of (2.14) are stable if and only if $y_{1}(T)+\dot{y}_{2}(T)$ is real and either

$$
\left|y_{1}(T)+\dot{y}_{2}(T)\right|<2
$$

or

$$
y_{1}(T)+\dot{y}_{2}(T)= \pm 2 \text { and } y_{2}(T)=\dot{y}_{1}(T)=0 .
$$

If in (2.14) the function $Q(t)$ is even, i. e. $Q(t)=Q(-t)$, it is possible to establish relations between the values of $y_{1}, \dot{y}_{1}, y_{2}$ and $\dot{y}_{2}$ at $t=T / 2$ and $t=T$ and these relations allow a more detailed classifications of the solutions of period $T$ and $2 T$.

Theorem 5. Let $y_{1}(t)$ and $y_{2}(t)$ be normalized solutions of (2.14), with $P(t) \equiv 1$ and $Q(t)$ even. Then the following relations hold:

1. $y_{1}(T)=2 y_{1}(T / 2) \dot{y}_{2}(T / 2)-1=1+2 y_{2}(T / 2) \dot{y}_{1}(T / 2)$;

2. $y_{2}(T)=2 y_{2}(T / 2) \dot{y}_{2}(T / 2)$;

3. $\dot{y}_{1}(T)=2 y_{1}(T / 2) \dot{y}_{1}(T / 2)$;

4. $\dot{y}_{2}(T)=y_{1}(T)$.

In all cases, $y_{1}(t)$ and $y_{2}(t)$ are the even and the odd solution of (2.14). Whenever a non-trivial solution of period $T$ or $2 T$ exists, there also exists such a solution which is either odd or even. Therefore, these periodic solutions are necessarily multiples of one of the normalized solutions $y_{1}(t)$ or $y_{2}(t)$ unless all solutions are periodic (with period $T$ or $2 T$ ).

Theorem 6. If the conditions of theorem above are satisfied, then there exists a nontrivial periodic solution of (2.14) which is

- Even and of period $T$ if and only if $\dot{y}_{1}(T / 2)=0$;

- odd and of period $T$ if and only if $y_{2}(T / 2)=0$;

- even and of period $2 T$ if and only if $y_{1}(T / 2)=0$;

- odd and of period $2 T$ if and only if $\dot{y}_{2}(T / 2)=0$.

Periodic solutions of period $T$ or $2 T$ are necessarily multiples of the normalized solutions $y_{1}(t)$ and $y_{2}(t)$. 


\subsection{Elementary Properties of Real Analytic functions}

In this section we'll remember some definitions and properties of analytic functions on the real line, which we'll use in the next chapter.

We begin with power series on the real line $\mathbb{R}$

$$
\sum_{k=0}^{\infty} a_{k}(x-\alpha)^{k},
$$

where the $a_{k}^{\prime} s$ being either real or complex constants.

Let the power series (2.19) convergent at $x=c$, and $r=|c-\alpha|$. Then the series converges uniformily and absolutely on compact subsets of $X=\{x:|x-c|<r\}$.

We call Interval of Convergence of the power series (2.19), the interval centered about $\alpha$ and radius defined to be half its length.

Let a power series (2.19) with interval of convergence $\mathcal{C}$, we can define a function $f$ on $\mathcal{C}=\mathcal{C}_{f}$, and such function is said to be real analytic at $\alpha$.

Definition. A function $f$, with domain an open set $U \subset \mathbb{R}$, and range either real line or complex plane, is said to be Real Analytic at $\alpha$ if the function may be represented by a convergent power series on some interval of positive radius centered at $\alpha$ :

$$
f(x)=\sum_{k=0}^{\infty} a_{k}(x-\alpha)^{k} .
$$

The function is said to be Real Analytic on $V \subset U$ if it is real analytic at each $\alpha \in V$.

Some of properties of real analytic function will be resumed in next propositions.

Proposition 6. Let

$$
\sum_{k} a_{k}(x-\alpha)^{k} \text { and } \sum_{k} b_{k}(x-\alpha)^{k}
$$

be two power series which define the functions $f(x)$ and $g(x)$ on open intervals of convergence $\mathcal{C}_{f}$ and $\mathcal{C}_{g}$ respectively. Then on their common domain $\mathcal{C}=\mathcal{C}_{f} \cap \mathcal{C}_{g}$ we have

- $f(x) \pm g(x)=\sum_{k}\left(a_{k} \pm b_{k}\right)(x-\alpha)^{k}$;

- $f(x) g(x)=\sum_{m=0}^{\infty} \sum_{j+k=m}\left(a_{j} b_{k}\right)(x-\alpha)^{m}$;

- If $g(x)$ does not vanish on $I \subset \mathcal{C}$ centered at $\alpha$, then the function

$$
h(x)=\frac{f(x)}{g(x)}
$$

is real analytic at $\alpha$.

Remark 4. The power series expansion of $h(x)=\sum_{k} c_{k}(x-\alpha)^{k}$ on I may be obtained as following:

$$
\begin{gathered}
c_{0}=\frac{a_{0}}{b_{0}} ; \\
c_{1}=\frac{1}{b_{0}}\left(a_{1}-\frac{a_{0} b_{1}}{b_{0}}\right) ; \\
c_{k}=\frac{1}{b_{0}}\left(a_{k}-b_{1} c_{k-1}\right) .
\end{gathered}
$$


Proposition 7. (Hadamard Formula): For the power series

$$
\sum_{k} a_{k}(x-\alpha)^{k}
$$

define $A$ and $\rho$ by

$$
\begin{gathered}
A=\lim _{n \rightarrow \infty} \sup _{\left|a_{n}\right|^{1 / n}} \\
\rho=\left\{\begin{array}{c}
0, \text { if } A=\infty \\
1 / A, \text { if } 0<A<\infty \\
\infty, \text { if } A=0
\end{array}\right.
\end{gathered}
$$

then $\rho$ is the radius of convergence of the power series about $\alpha$

One consequence of the proposition above that we use forward is

Corollary 2. The power series

$$
\sum_{k} a_{k}(x-\alpha)^{k}
$$

has radius of convergence $\rho$ if and only if, for each $0<r<\rho$, there exists a constant $0<C=C_{r}$ such that

$$
\left|a_{k}\right| \leq \frac{C}{r^{k}}
$$

Proposition 8. The radius of convergence of the derived series is the same as the radius of convergence of the original power series.

When we pass to function we have

Proposition 9. Let

$$
\sum_{k} a_{k}(x-\alpha)^{k}
$$

be a power series with $f(x)$ be the function defined by its on an open interval of convergence $\mathcal{C}_{f}$. Then $f$ is continuous and has continuous derivatives of all orders which are real analytic at $\alpha$.

This implies that a real analytic function has a unique power series representation

Corollary 3. If the function $f$ is represented by a convergent power series on an interval of positive radius centered at $\alpha$,

$$
f(x)=\sum_{k} a_{k}(x-\alpha)^{k},
$$

then the coefficients of the power series are related to the derivatives of the function by

$$
a_{k}=\frac{f^{(k)}(\alpha)}{k !}
$$

Corollary 4. If $f \in \mathcal{C}^{\omega}(I)$ for some open interval I then, for each $\alpha \in I$, there are an open interval $J$, with $\alpha \in J \subset I$, and constants $C>0$ and $r>0$ such that the derivatives of $f$ satisfy

$$
\left|f^{(k)}(x)\right| \leq C \frac{k !}{r^{k}}, \quad \forall x \in J .
$$

Corollary 5. If $f \in \mathcal{C}^{\infty}(I)$ for some open interval $I$ and if, for each $\alpha \in I$, there are an open interval $J$, with $\alpha \in J \subset I$, and constants $C>0$ and $r>0$ such that the derivatives of $f$ satisfy

$$
\left|f^{(k)}(x)\right| \leq C \frac{k !}{r^{k}}, \quad \forall x \in J,
$$

then $f \in \mathcal{C}^{\omega}(I)$. 
The two corollaries can merge into

Theorem 7. Let $f \in \mathcal{C}^{\infty}(I)$ for some open interval I. The function $f$ is in $\mathcal{C}^{\omega}(I)$ if and only if, for each $\alpha \in I$, there are an open interval $J$, with $\alpha \in J \subset I$, and constants $C>0$ and $r>0$ such that the derivatives of $f$ satisfy

$$
\left|f^{(k)}(x)\right| \leq C \frac{k !}{r^{k}}, \quad \forall x \in J .
$$

Now we will give the definition of Newton's Binomial.

Definition. Let $x \in \mathbb{C}$ and $\beta \in \mathbb{C}$. We define

$$
(1+x)^{\beta}=\sum_{k=0}^{\infty}\left(\begin{array}{l}
\beta \\
k
\end{array}\right) x^{k},
$$

where

$$
\left(\begin{array}{l}
\beta \\
k
\end{array}\right)=\frac{\beta(\beta-1)(\beta-2) \ldots(\beta-k+1)}{k !}
$$




\section{Chapter 3}

\section{Planar Positional Force Fields}

\subsection{Introduction}

In this chapter, we will introduce the definition of a planar positional force field, or Newton systems. Zampieri in [37] raised a remark about how little we know on this subject, since it is not much studied as we study conservative systems or vector fields in the plane. Indeed, an explanation for this maybe is because in conservative case the evolution of geometries (Symplectic, Riemannian, ...) showed more interesting for mathematicians and besides that, most of us prefer to study this problem with a point of view of vector fields in $\mathbb{R}^{4}$ and not a force field in $\mathbb{R}^{2}$.

In fact there is some very important in this subject if we date back to Newton's Principia (Kepler's problem is a planar problem). We can quote others works as Appell [3, 4], Bertrand [11, 12, 13], Darboux [14], Halphen [17], Lagrange [26], Laplace [31], more recently Kasner [19, 20, 21, 22, 23], Albouy [1, 2], Barone et al [8, 9, 15, 16] and Zampieri et al [37, 38]. Most of those works are about first integral for positional system except the works of Kasner which he studied the problem with the point of view of planar curves. The works of Barone et al and Zampieri et al went in a way to study stability for this type of system.

Indeed we may think this system as a problem of second order ordinary differential equation, as Arnold observed [5], second order ODE is mechanics. On first integral, we will return to this subject in the next chapter, here we introduce the definition, a point transformation for positional systems.

Consider the motion of a particle in the plane under the action of any positional field force, that is the force depends only on position of the particle. The general equations of motion is given by

Definition. A planar positional system of one particle, is an equation of the form (2.6), where $\boldsymbol{x}=(x, y)$ and $\boldsymbol{F}(\boldsymbol{x}, \boldsymbol{v}, t)=\boldsymbol{F}(\boldsymbol{x})=(X(\boldsymbol{x}), Y(\boldsymbol{x}))$. Thus, we have

$$
\left\{\begin{array}{c}
\frac{d^{2} x}{d t^{2}}-X(x, y)=0 \\
\frac{d^{2} y}{d t^{2}}-Y(x, y)=0
\end{array}\right.
$$

with the reference origin being the point $(x, y)=(0,0)$. For further purpose, we suppose that $X(x, y)$ and $Y(x, y)$ are smooth functions in some open neighborhood of the $(0,0)$, thus $X(x, y), Y(x, y) \in$ $\mathcal{C}^{k}(\Omega), \Omega=\Omega^{\circ} \subset \mathbb{R}^{2}$.

Probably, the most celebrated work which is strictly positional is the Kepler's problem. One point mass is attracted by a fixed point in a plane, and it describes a conic. Some references about this are $[17,27,36]$. Another example where the force field is positional only is the Two point fixed problem (or Euler's three-body problem), which we will illustrate the method given by Lagrange [26], since it is the starting point of a systematic study about first integral of a general planar positional force field.

In his beautiful work, "Recherches sur le mouvement d'un corps qui est attiré vers deux centres fixes", Lagrange given a more elegant proof of a problem discussed by Euler (Nov. Comm. Acad. 
Imp. Petropolitanae, 10, pp. $207-242$ ), which is to solve for the motion of a particle that is acted upon by the gravitational field of two other point masses that are fixed in space. The difference between the two proofs is that the Lagrange's proof did not impose any restriction on the motion of the particle studied.

Lagrange divided his work into two mémoire: In the first one, he used as the force field the gravitational Newtonian force law. He proved the integrability of the problem by reduction the degrees of the system using the first integral known. In the second one, he searched for others law of attraction such that the problem is still integrable. Since the first argument for integrability was the reduction by first integral then he studied systemically first integral which are quadratic in velocities and find Laws of attraction where the system had these first integral as a constant of motion.

Euler's Three-body Problem. Let $\tilde{x}, \tilde{y}, \tilde{z}$ rectangular coordinates of the particle. Let $x_{i}, y_{i}, z_{i}$, $i \in 1,2$ the rectangular coordinates for the two masses fixed. Define the distances

$$
\rho_{i}=\left[\left(\tilde{x}-x_{i}\right)^{2}+\left(\tilde{y}-y_{i}\right)^{2}+\left(\tilde{z}-z_{i}\right)^{2}\right]^{1 / 2} .
$$

Let us write the attraction law as $F_{i}, i \in 1,2$, with $F_{i}=F_{i}\left(\rho_{i}\right)$. Then the equations of motion of the particles are

$$
\left\{\begin{array}{l}
\frac{d^{2} \tilde{x}}{d t^{2}}+\sum_{i=1,2} \frac{\left(\tilde{x}-x_{i}\right) F_{i}}{\rho_{i}}=0 \\
\frac{d^{2} \tilde{y}}{d t^{2}}+\sum_{i=1,2} \frac{\left(\tilde{y}-y_{i}\right) F_{i}}{\rho_{i}}=0 \\
\frac{d^{2} \tilde{z}}{d t^{2}}+\sum_{i=1,2} \frac{\left(\tilde{z}-z_{i}\right) F_{i}}{\rho_{i}}=0
\end{array}\right.
$$

We have as a constant of motion

$$
\dot{\tilde{x}}^{2}+\dot{\tilde{y}}^{2}+\dot{\tilde{z}}^{2}+2 \int F_{1} d \rho_{1}+2 \int F_{2} d \rho_{2},
$$

provided the forces are functions $F_{i}=F_{i}\left(\rho_{i}\right)$.

Let the distance between the two fixed masses be

$$
\delta=\left[\left(x_{1}-x_{2}\right)^{2}+\left(y_{1}-y_{2}\right)^{2}+\left(z_{1}-z_{2}\right)^{2}\right]^{1 / 2} .
$$

If we multiply the first equation of (3.2) by $\left(\tilde{x}-x_{1}\right)$, the second by $\left(\tilde{y}-y_{1}\right)$ and the third one by $\left(\tilde{z}-z_{1}\right)$ and sum up the result, we arrive with

$$
\frac{1}{2} \frac{d^{2}}{d t^{2}}\left(\rho_{1}^{2}\right)+F_{1} \rho_{1}+\frac{\rho_{1}^{2}+\rho_{2}^{2}-\delta^{2}}{2 \rho_{2}} F_{2}+2 \int F_{1} d \rho_{1}+2 \int F_{2} d \rho_{2}=0
$$

and the same method, but now we multiply by $\left(\tilde{x}-x_{2}\right),\left(\tilde{x}-y_{2}\right)$ and $\left(\tilde{x}-z_{2}\right)$, and we arrive

$$
\frac{1}{2} \frac{d^{2}}{d t^{2}}\left(\rho_{2}^{2}\right)+F_{2} \rho_{2}+\frac{\rho_{1}^{2}+\rho_{2}^{2}-\delta^{2}}{2 \rho_{1}} F_{1}+2 \int F_{1} d \rho_{1}+2 \int F_{2} d \rho_{2}=0 .
$$

To simplify, let us write

$$
\rho_{1}^{2}=\delta^{2} x, \quad \rho_{2}^{2}=\delta^{2} y, \quad \frac{F_{1}}{\rho_{1}}=M, \quad \frac{F_{2}}{\rho_{2}}=N,
$$


then we have

$$
\left\{\begin{array}{l}
\frac{1}{2} \frac{d^{2} x}{d t^{2}}+M x+\frac{x+y-1}{2} N+\int M d x+\int N d y=0 \\
\frac{1}{2} \frac{d^{2} y}{d t^{2}}+N y+\frac{x+y-1}{2} M+\int M d x+\int N d y=0
\end{array}\right.
$$

where $M$ is a function of $x$ and $N$ is a function of $y$, which is of form (3.1).

So Lagrange transformed a system tridimensional to one planar and positional like (3.1).

One of the Newton's law is the inertial quality of the equation (2.6) which is expressed on the left-hand side in the form "mass times acceleration" or "rate of change of the momentum." In other hand the principles of analytical mechanics have shown that the really fundamental quantity which characterizes the inertia of mass is not momentum but the kinetic energy. This kinetic energy is a scalar quantity and defined as $\frac{1}{2} m<\mathbf{v}, \mathbf{v}>$ for a single particle, where $<$, $>$ is the usual scalar product and $m$ the mass of particle.

We already shown that from the point of the view of Euclidean geometry, no choice of $(l, m, n)$, satisfying $l n-m^{2}>0$ in (2.4), is distinguished. So do our principles of analytical mechanics. This means that if we define a "new" metric (scalar product) we want that the "new" kinetic energy, for a constant mass, satisfy the same principles.

Let

$$
A=\left(\begin{array}{ll}
\alpha & \gamma \\
\gamma & \beta
\end{array}\right), \alpha, \beta, \gamma \in \mathcal{C}^{2}(\Omega, \mathbb{R})
$$

be positive definite on the simply connected open domain $\Omega$. Let us define the scalar product $<<;>>$ by

$$
<\left\langle\mathbf{u} ; \mathbf{v}>>=<\mathbf{u} ; A \mathbf{v}>=\alpha u_{1} v_{1}+\gamma\left(u_{1} v_{2}+u_{2} v_{1}\right)+\beta u_{2} v_{2},\right.
$$

where $\mathbf{u}=\left(u_{1}, u_{2}\right), \mathbf{v}=\left(v_{1}, v_{2}\right)$, and $<;>$ is the usual scalar product. The Kinetic energy $T$ defined by $<<;>>$ is

$$
T=\frac{m}{2}<<\mathbf{v} ; \mathbf{v}>>=\frac{1}{2}\left(\alpha \dot{x}^{2}+2 \gamma \dot{x} \dot{y}+\beta \dot{y}^{2}\right)
$$

for a material point of mass $m$ and $\mathbf{v}=(\dot{x}, \dot{y})$.

Let us begin with the equation (2.6), with $\mathbf{F}(\mathbf{x}, \mathbf{v}, t)=\mathbf{F}(\mathbf{x})$. Thus if we take the scalar product with the velocity to both side, we have

$$
\left\langle\frac{d(m \mathbf{v})}{d t}, \mathbf{v}\right\rangle=\langle\mathbf{F}, \mathbf{v}\rangle
$$

and the left side is equal to $\frac{1}{2} \frac{d}{d t} m\langle\mathbf{v}, \mathbf{v}\rangle=\frac{d}{d t} T_{0}, T_{0}$ is the kinetic energy with usual scalar product. Thus we have

$$
\frac{d}{d t} T_{0}=<\mathbf{F}, \mathbf{v}>
$$

In the new metric we want the same principles, so

$$
\frac{d}{d t} T=\frac{d}{d t} \frac{m}{2}<<\mathbf{v} ; \mathbf{v}>>=<<\mathbf{F}, \mathbf{v}>>,
$$

but the last equality is true if and only if

$$
\left\langle\mathbf{v},\left(\frac{d A}{d t}\right) \mathbf{v}\right\rangle=0
$$

which is true if we have $\alpha(x, y), \beta(x, y), \gamma(x, y)$ satisfying the following system of partial differential equations

$$
\left\{\frac{\partial \alpha}{\partial x}=\frac{\partial \beta}{\partial y}=0, \quad \frac{1}{2} \frac{\partial \alpha}{\partial y}+\frac{\partial \gamma}{\partial x}=0, \quad \frac{1}{2} \frac{\partial \beta}{\partial x}+\frac{\partial \gamma}{\partial y}=0\right.
$$


This yields

$$
\left\{\begin{array}{c}
\alpha=a+b y+c y^{2} \\
\beta=g+h x+c x^{2} \\
\gamma=k-\frac{1}{2} b x-\frac{1}{2} h y-c x y
\end{array}\right.
$$

where $a, b, c, g, h, k$ are constants.

Those equations (3.10) was found by Lagrange, and after him by Laplace and Bertrand. In their works, they've looked for a first integral of the system (3.1) which has the form

$$
V(x, y, \dot{x}, \dot{y})=\alpha(x, y) \dot{x}^{2}+2 \gamma(x, y) \dot{x} \dot{y}+\beta(x, y) \dot{y}^{2}+\pi(x, y) .
$$

We can resume the results by Lagrange and the results by Barone and Mauro in the next proposition

Proposition 10. Let $V=V(x, y, \dot{x}, \dot{y}): \mathbb{R}^{4} \rightarrow \mathbb{R}$ be a first integral for a system (3.1), quadratic in velocities with coefficients, functions of $(x, y)$, that is

$$
V(x, y, \dot{x}, \dot{y})=\alpha(x, y) \dot{x}^{2}+2 \gamma(x, y) \dot{x} \dot{y}+\beta(x, y) \dot{y}^{2}+\pi(x, y) .
$$

Then, the velocity part of $V(x, y, \dot{x}, \dot{y})$

$$
\alpha(x, y) \dot{x}^{2}+2 \gamma(x, y) \dot{x} \dot{y}+\beta(x, y) \dot{y}^{2},
$$

is a quadratic form in the variables $\dot{x}, \dot{y}, x \dot{y}-y \dot{x}$ with constants coefficients. Moreover, $(\alpha X+$ $\gamma Y) d x+(\gamma X+\beta Y) d y$, where $X(x, y), Y(x, y)$ is the force field, is an exact differential one-form.

Proof. Let us begin with a quadratic and homogeneous functions in velocities

$$
V(x, y, \dot{x}, \dot{y})=\alpha(x, y) \dot{x}^{2}+\beta(x, y) \dot{y}^{2}+2 \gamma(x, y) \dot{x} \dot{y}+\pi(x, y) .
$$

By hypothesis $V$ is a first integral of (3.1), then $\frac{d}{d t} V$ give us

$$
\begin{gathered}
\left\{\begin{array}{c}
\frac{\partial \alpha}{\partial x}=\frac{\partial \beta}{\partial y}=0 \\
\frac{1}{2} \frac{\partial \alpha}{\partial y}+\frac{\partial \gamma}{\partial x}=0 \\
\frac{1}{2} \frac{\partial \beta}{\partial x}+\frac{\partial \gamma}{\partial y}=0
\end{array}\right. \\
\left\{\begin{array}{l}
\alpha X+\gamma Y+\pi_{x}=0 \\
\gamma X+\beta Y+\pi_{y}=0,
\end{array}\right.
\end{gathered}
$$

And the first system above is equal that we obtained before, we still have the same result in (3.10). Thus, the quadratic part of $V$ is

$$
\left(a+b y+c y^{2}\right) \dot{x}^{2}+\left(g+h x+c x^{2}\right) \dot{y}^{2}+\left(k-\frac{1}{2} b x-\frac{1}{2} h y-c x y\right) \dot{x} \dot{y},
$$

which can be written as

$$
a \dot{x}^{2}+g \dot{y}^{2}+2 k \dot{x} \dot{y}+b \dot{x}(y \dot{x}-x \dot{y})+h \dot{y}(x \dot{y}-y \dot{x})+c(y \dot{x}-x \dot{y})^{2} .
$$

The moreover part is directly from the third system above, since

$$
d(-\pi)=(\alpha X+\gamma Y) d x+(\gamma X+\beta Y) d y
$$


For the positional planar problem (3.1) this quadratic first integral plays an important role to stability theory, like the Hamiltonian function (energy first integral) does for conservative forces. Thus it is fair enough we dedicate a part of this thesis to illustrated how it is important.

As the importance of the potential function has to conservative system, we can define a special class of system which has a special function, namely pseudo-potential, which plays the same role of potential functions for conservative system. Let us define,

Definition. If exists $\pi: \Omega \rightarrow \mathbb{R}, \pi \in \mathcal{C}^{2}$, such that

$$
-\pi_{x}=\alpha X+\gamma Y, \quad-\pi_{y}=\gamma X+\beta Y,
$$

then we say that the force $F=(X, Y)$ is pseudo-conservative, or quasi-potential. We call the function $\pi$ the pseudo-potential for the force.

The following property comes as a connection between the definition of pseudo-conservative forces and the existence of quadratic first-integral.

Proposition 11. The force $F=(X, Y)$ is pseudo-conservative (for some metric) if and only if there exist $a, b, c, g, h, k \in \mathbb{R}$ such that $A$ defined by (3.6) with (3.10) is positive definite and

$$
\begin{gathered}
\frac{3}{2}(b+2 c y) X-\frac{3}{2}(h+2 c x) Y+\left(k-\frac{1}{2} b x-\frac{1}{2} h y-c x y\right)\left(Y_{y}-X_{x}\right) \\
+\left(a+b y+c y^{2}\right) X_{y}-\left(g+h x+c x^{2}\right) Y_{x}=0 .
\end{gathered}
$$

The next two proposition show the importance of pseudo-conservative forces, since, in this case, we can show the existence of a Lyapunov functions for the system and derive a Dirichlet-Lagrange like theorem for pseudo-conservative systems.

Proposition 12. Under the condition of proposition (11), the function (3.18) is a first integral for the system (3.1). (Conservation of mechanical energy with respect to the new metric.)

Proposition 13. The map $V$ in (3.18) is a first integral of (4.1) if and only if (3.14) holds for some $a, b, c, g, h, k \in \mathbb{R}$.

Remark 5. The matrix A, defined by (3.6) with (3.10), is not necessarily positive definite, it was in proposition (11). This is the difference between propositions (11) and (13)

And we arrive in the following definition for the pseudo-conservative force field

$$
\begin{aligned}
& X=\frac{\gamma \frac{d \pi}{d y}-\beta \frac{d \pi}{d x}}{\alpha \beta-\gamma^{2}} \\
& Y=\frac{\gamma \frac{d \pi}{d x}-\alpha \frac{d \pi}{d y}}{\alpha \beta-\gamma^{2}}
\end{aligned}
$$

One remark we observe from these equations is that $\pi$ is not the "potential" of the force, but if we assume $\alpha$ and $\beta$ constant and $\gamma \equiv 0$ we indeed have $\pi$ as a potential of the force $(X, Y)$, and we call this as conservative force field.

Let us define $\alpha \beta-\gamma^{2}=\Delta$ and $\pi=\Delta^{2} \Pi$, where $\Pi$ is some function of $(x, y)$. If we put this definition into (3.15), we arrive

$$
\begin{aligned}
& X=\Delta\left(\Pi \frac{d \beta}{d x}+2 \beta \frac{d \Pi}{d x}-\gamma \frac{d \Pi}{d y}\right) \\
& Y=\Delta\left(\Pi \frac{d \alpha}{d y}+2 \alpha \frac{d \Pi}{d y}-\gamma \frac{d \Pi}{d x}\right)
\end{aligned}
$$

where

$$
\Delta=\left(a g-k^{2}\right)+(a h+b k) x+(b g+h k) y+\left(a c-\frac{1}{4} b^{2}\right) x^{2}+\left(c g-\frac{1}{4} h^{2}\right) y^{2}+\left(\frac{1}{2} b h+2 c k\right) x y .
$$


Thus for the system (3.1), when the force is pseudo-conservative, we have the following first integral

$$
\alpha(x, y) \dot{x}^{2}+2 \gamma(x, y) \dot{x} \dot{y}+\beta(x, y) \dot{y}^{2}+\Delta^{2} \Pi=V(x, y, \dot{x}, \dot{y}) .
$$

\subsection{Point Transformations on Planar Positional Force}

Let any positional field of force in the plane, with corresponding equations of motion (3.1), an arbitrary coordinate transformation, unaccompanied by any change in the time, will leads to a new differential equations which it will in general involve not only the new position $x_{1}, y_{1}$ but also the velocities $\frac{d x_{1}}{d t}$ and $\frac{d y_{1}}{d t}$. In fact, the only exception is where the transformation is merely affine: $\varphi(x, y)=a_{1} x+b_{1} y+c_{1}, \psi(x, y)=a_{2} x+b_{2} y+c_{2}$.

Indeed, Appell in 1889 brought out a coordinate transformation where the new system is still a positional one, this is a generalization that Halphen had obtained before, with the projective transformations, that is

Proposition 14. (Appell, [3]): The coordinate (or point) transformation which takes a positional system (3.1) and leads it to another positional system are the homographies, with respective time transformation. In other words

$$
\left\{\begin{aligned}
\frac{d^{2}}{d t_{1}^{2}} \varphi(x, y) & =X_{1}(\varphi, \psi) \\
\frac{d^{2}}{d t_{1}^{2}} \psi(x, y) & =Y_{1}(\varphi, \psi)
\end{aligned}\right.
$$

where

$$
\varphi(x, y)=\frac{a_{1} x+b_{1} y+c_{1}}{a_{3} x+b_{3} y+c_{3}}, \quad \psi(x, y)=\frac{a_{2} x+b_{2} y+c_{2}}{a_{3} x+b_{3} y+c_{3}}, \quad \kappa d t_{1}=\frac{d t}{\left(a_{3} x+b_{3} y+c_{3}\right)^{2}},
$$

and $k$ is an arbitrary constant (constant of integration) and the coefficients $a_{i}^{\prime} s, b_{i}^{\prime} s, c_{i}^{\prime} s$ are constants and the determinant of

$$
M=\left(\begin{array}{lll}
a_{1} & b_{1} & c_{1} \\
a_{2} & b_{2} & c_{2} \\
a_{3} & b_{3} & c_{3}
\end{array}\right),
$$

does not vanish.

With the new field given by

$$
\begin{gathered}
X_{1}=k^{2}\left(a_{3} x+b_{3} y+c_{3}\right)^{2}\left\{C_{2}(x Y-y X)+B_{2} X-A_{2} Y\right\} \\
Y_{1}=k^{2}\left(a_{3} x+b_{3} y+c_{3}\right)^{2}\left\{-C_{1}(x Y-y X)-B_{1} X+A_{1} Y\right\},
\end{gathered}
$$

and the inverse

$$
\begin{gathered}
x=\frac{A_{1} \varphi+A_{2} \psi+A_{3}}{C_{1} \varphi+C_{2} \psi+C_{3}}, y=\frac{B_{1} \varphi+B_{2} \psi+B_{3}}{C_{1} \varphi+C_{2} \psi+C_{3}}, \\
a_{3} x+b_{3} y+c_{3}=\frac{\operatorname{det}(M)}{C_{1} \varphi+C_{2} \psi+C_{3}}
\end{gathered}
$$

where the capital letters are the co-factors determinant to its respective minors letters.

For simplicity let us fix as definition $x_{1}(t)=\varphi(x(t), y(t))$ and $y_{1}(t)=\psi(x(t), y(t))$.

The velocities in this new coordinates is given by

$$
\begin{gathered}
\dot{x}_{1}=\frac{d x_{1}}{d t_{1}}=\left(\frac{\partial x_{1}}{\partial x} \dot{x}+\frac{\partial x_{1}}{\partial y} \dot{y}\right) \frac{d t}{d t_{1}} \\
=\left\{\left(\frac{-C_{2} y+B_{2}}{\left(a_{3} x+b_{3} y+c_{3}\right)^{2}}\right) \dot{x}+\left(\frac{C_{2} x-A_{2}}{\left(a_{3} x+b_{3} y+c_{3}\right)^{2}}\right) \dot{y}\right\} k\left(a_{3} x+b_{3} y+c_{3}\right)^{2}
\end{gathered}
$$




$$
=k\left\{C_{2}(x \dot{y}-y \dot{x})+B_{2} \dot{x}-A_{2} \dot{y}\right\},
$$

and

$$
\begin{gathered}
\dot{y}_{1}=\frac{d y_{1}}{d t_{1}}=\left(\frac{\partial y_{1}}{\partial x} \dot{x}+\frac{\partial y_{1}}{\partial y} \dot{y}\right) \frac{d t}{d t_{1}} \\
=\left\{\left(\frac{C_{1} y-B_{1}}{\left(a_{3} x+b_{3} y+c_{3}\right)^{2}}\right) \dot{x}+\left(\frac{-C_{1} x+A_{1}}{\left(a_{3} x+b_{3} y+c_{3}\right)^{2}}\right) \dot{y}\right\} k\left(a_{3} x+b_{3} y+c_{3}\right)^{2} \\
=k\left\{-C_{1}(x \dot{y}-y \dot{x})-B_{1} \dot{x}+A_{1} \dot{y}\right\},
\end{gathered}
$$

A consequence of the proposition is applied on a particular case of (3.1), that is, the following system

$$
\left\{\begin{array}{l}
\ddot{x}=-x f(x) \\
\ddot{y}=-y f(x), \quad(x, y) \in \Omega=\Omega^{\circ} \subset \mathbb{R}^{2}, 0 \in \Omega,
\end{array}\right.
$$

which we study the stability afterward.

Corollary 6. The only point transformations that preserve a given system (3.20) are

$$
x_{1}=\frac{a_{1} x}{a_{3} x+c_{3}}, \quad y_{1}=\frac{a_{2} x+b_{2} y}{a_{3} x+c_{3}},
$$

with the change of time be

$$
d t_{1}=\frac{\bar{k} d t}{\left(a_{3} x+c_{3}\right)^{2}}
$$

where preserve means that the system is still positional with $\ddot{x}_{1}=G\left(x_{1}\right)$ and (in this case) take the origin to the new origin $(x, y)=(0,0) \rightarrow\left(x_{1}, y_{1}\right)=(0,0)$. Moreover, we have

$$
\left\{\begin{array}{l}
\ddot{x}_{1}=-x_{1} g\left(x_{1}\right) \\
\ddot{y}_{1}=-y_{1} g\left(x_{1}\right), \quad\left(x_{1}, y_{1}\right) \in \Omega_{1}=\Omega_{1}^{\circ} \subset \mathbb{R}^{2}, 0 \in \Omega_{1},
\end{array}\right.
$$

where the dot for the last system means derivative with respect to $t_{1}$ and

$$
g\left(x_{1}\right)=\frac{k}{\left(a_{1}-a_{3} x_{1}\right)^{3}}\left(f \circ x\left(x_{1}\right)\right) .
$$

Proof. The proof for this fact is same as the proof of Appell for the general planar postional system. Let us begin with the problem: Find a point transformation, with the respective change in time

$$
x_{1}=\varphi(x, y), y_{1}=\psi(x, y), d t_{1}=\lambda(x, y) d t
$$

such that the system (3.20) is preserved in the sense of our hypothesis. Take the first derivative of $x_{1}$ (for the $y_{1}$ the proof is equal, so we only do the $x_{1}$ case):

$$
\begin{gathered}
\frac{d x_{1}}{d t_{1}}=\frac{d x_{1}}{d t} \frac{d t}{d t_{1}}=\left\{\frac{\partial \varphi}{\partial x} \dot{x}+\frac{\partial \varphi}{\partial y} \dot{y}\right\} \frac{1}{\lambda}, \\
\frac{d^{2} x_{1}}{d t_{1}^{2}}=\frac{d}{d t}\left\{\left[\frac{\partial \varphi}{\partial x} \dot{x}+\frac{\partial \varphi}{\partial y} \dot{y}\right] \frac{1}{\lambda}\right\} \\
=\left\{\frac{1}{\lambda^{2}} \frac{\partial \varphi}{\partial x} \ddot{x}+\frac{1}{\lambda^{2}} \frac{\partial \varphi}{\partial y} \ddot{y}\right\}+\left\{\frac{1}{\lambda} \frac{\partial}{\partial x}\left(\frac{1}{\lambda} \frac{\partial \varphi}{\partial x}\right)\right\} \dot{x}^{2}+\left\{\frac{1}{\lambda} \frac{\partial}{\partial y}\left(\frac{1}{\lambda} \frac{\partial \varphi}{\partial y}\right)\right\} \dot{y}^{2} \\
+\left\{\frac{\partial}{\partial y}\left(\frac{1}{\lambda} \frac{\partial \varphi}{\partial x}\right)+\frac{\partial}{\partial x}\left(\frac{1}{\lambda} \frac{\partial \varphi}{\partial y}\right)\right\}\left(\frac{1}{\lambda}\right) \dot{x} \dot{y} .
\end{gathered}
$$

For the system (3.20) to be preserved we imposed that all coefficients which multiply the veloc- 
ities terms must vanish (the system of partial differential equations is the same of Appell's proof). Beside them, we want that the term which multiplies $\ddot{y}$ also vanish $\left(\ddot{x}_{1}=G\left(x_{1}\right)\right)$. So we have an additional constraint

$$
\frac{\partial \varphi}{\partial y}=0 \Rightarrow \varphi=\varphi(x) .
$$

And the solution of the system of PDE we arrive at

$$
\lambda(x)=\frac{\bar{k}}{\left(a_{3} x+c_{3}\right)^{2}} ; \quad \varphi(x)=\frac{a_{1} x+c_{1}}{a_{3} x+c_{3}} ; \quad \psi(x, y)=\frac{a_{2} x+b_{2} y+c_{2}}{a_{3} x+c_{3}} .
$$

To accomplished our hypothesis of preserved system $\left((x, y)=(0,0) \rightarrow\left(x_{1}, y_{1}\right)=(0,0)\right)$ we impose that $c_{1}=c_{2}=0$.

The second part of the proof. Since we can write $x$ as a function of $x_{1}$

$$
x=\frac{c_{3} x_{1}}{a_{1}-a_{3} x_{1}},
$$

and we have $\ddot{x}_{1}=\frac{1}{\lambda^{2}} \frac{\partial \varphi}{\partial x} \ddot{x}$, we see that $X_{1}$ is only $x_{1}$ dependent and after substitution we arrive $X_{1}=-x_{1} \frac{c_{3} B_{2}^{3}}{k^{2}\left(a_{1}-a_{3} x_{1}\right)^{3}}(f \circ x)\left(x_{1}\right)$.

For the $Y_{1}$, it is not trivial to see that $Y_{1}=-y_{1} \frac{c_{3} B_{2}^{3}}{k^{2}\left(a_{1}-a_{3} x_{1}\right)^{3}}(f \circ x)\left(x_{1}\right)$, since $y$ is a function of not only $y_{1}$ but also $x_{1}$ and

$$
\begin{gathered}
\ddot{y}_{1}=\frac{1}{\lambda^{2}} \frac{\partial \psi}{\partial x} \ddot{x}+\frac{1}{\lambda^{2}} \frac{\partial \psi}{\partial y} \ddot{y} . \\
\frac{\partial \psi}{\partial x}=\frac{\left(a_{2} c_{3}-a_{3} c_{2}\right)-a_{3} b_{2} y}{\left(a_{3} x+c_{3}\right)^{2}}, \\
\frac{\partial \psi}{\partial y}=\frac{b_{2}}{a_{3} x+c_{3}}, \\
y=\frac{1}{b_{2}}\left[\frac{B_{1} x_{1}+B_{2} y_{1}+B_{3}}{a_{1}-a_{3} x_{1}}\right] .
\end{gathered}
$$

After substitution we arrive

$$
\begin{gathered}
\ddot{y}_{1}=\left(-\frac{B_{2}^{4}}{\bar{k}^{2}\left(a_{1}-a_{3} x_{1}\right)^{4}}\right)\left(-\frac{c_{3} y_{1}\left(a_{1}-a_{3} x_{1}\right)}{B_{2}}\right)(f \circ x)\left(x_{1}\right) \\
=-y_{1} \frac{c_{3} B_{2}^{3}}{\bar{k}^{2}\left(a_{1}-a_{3} x_{1}\right)^{3}}(f \circ x)\left(x_{1}\right) .
\end{gathered}
$$

And we conclude the proof.

For now on, to simplify, we use the dot above $x, y$ to indicate the derivative with respect to $t\left({ }^{\cdot}=\frac{d}{d t}\right)$ and dot above $x_{1}, y_{1}$ to indicate the derivative with respect to $t_{1}\left(\cdot=\frac{d}{d t_{1}}\right)$. Another consequence of this proposition is: A solution of (3.19) is a homography transformation of a solution of (3.1). That is if a solution $\gamma(t)=(x(t), y(t))$ of (3.1) is a parametrized algebraic curve with degree $n$ then exist a solution $\bar{\gamma}(t)$ of (3.19) which is a parametrized algebraic curve with degree $n$ and $\bar{\gamma}(t)=\left(x_{1}(t), y_{1}(t)\right)$. This means that homography preserves the degree of an algebraic curve and if the curve is simple then preserve the convexity (or nonconvexity) of the set of the interior of the curve $\gamma(t)$.

Now is a good moment to discuss the difference between the Galilean transformations and the homographies since we presented both. Galilean transformations state the principle of mechanics, that is, all the law work for every location and time. So Galilean transformations is a map between two affine frames. In other hand, in each of this frames you can put a coordinates to study your problem, indeed if your problem is on a differentiable manifolds then there is an infinite choice of local charts, or local coordinates. Appell assured that for a planar positional problem, if you 
change the local coordinates by a homography accompanied with the change in time, your system is still a positional one, in the new coordinates. That is, the point transformation that preserves the dependence of position only by your system is given by a homography and the respective change in time. All other change of coordinate generated a system which depends on the velocity of the particle.

A remark we do about homographies is that a homography does not guarantee that the new system does not depend on the constraints in which the particle is subjected. That is, the condition $\left\langle\mathbf{v}, \frac{d A}{d t} \mathbf{v}\right\rangle=0$ does not preserve with a homography, this is direct from the fact that homography does not preserve the angle between vectors. 


\section{Chapter 4}

\section{Stability of Some Positional Systems}

Stability of the equilibrium is a mathematical field for two and a half century and one of its founder was Lagrange who stated the celebrated Lagrange-Dirichlet theorem in the $18^{\text {th }}$ century, which the so called converse of that statement is still unproven nowadays. So many mathematicians have been interested in stability and possible, the most important work in this subject is the doctoral thesis of Liapunov "The general problem of the stability of motion in 1892 [29]. The applications are also countless in mechanics and in most sciences. To start the study on this subject see Arnold [6], Meyer et al [32], Rouché et al, Kozlov et al [24], and the work of Liapunov [29].

The principal drawback of Liapunov's direct method is that no general procedure is known to construct auxiliary functions suiting specific theorems. That is why, in stability problems, one should a priori neglect no available information concerning the solutions. In particular, the first integrals will often be helpful, either to facilitate the search for auxiliary functions or to eliminate part of the variables and thus decrease the number of equations to examine.

About the positional planar problem (3.1), there is not many works about this matter. We can suggest Barone and Cesar [8], Zampieri and Barone [38], Zampieri [37], Cesar and Barone [15], Barone, Cesar and Gorni [9]. In this chapter we'll state some results about Liapunov stability for systems (3.1)

In the first section we state some new results about instability for the general problem, when $X=X(x, y)$ and $Y=Y(x, y)$. Basically, we use the first integrals found by Lagrange and linearized the equations of the system resultants at the origin to obtain the results. We will analyze the question of the stability of origin when we suppose $A$ in (3.6), is indefinite, that means $\Delta<0$ for $\alpha(x, y)>0$ in a neighborhood of the origin and we obtain a result like Dirichlet-Lagrange reverse, more precisely we prove that, suppose (3.1) which points inwards circumferences with center at the origin and it is pseudo-conservative with $A$ is an indefinite matrix function. If the pseudo-potential associated to $X, Y, \pi$, has a non-degenerated minimum at the origin, then it is a Liapunov unstable equilibrium.

After this, we prove a result for a certain type of those forces, which we call quasi-central attractor. We will give an example very useful where this result can apply and show how strong this result is.

The last section is dedicated for special type of central forces which are real analytics. We will show that, in the case of analytic forces, if we know the even part of the series of auxiliary potential associated to the force then we know all terms of the series.

\subsection{Systems With General Form}

\subsubsection{Introduction}

We consider the general system (3.1)

$$
\ddot{x}=X(x, y), \ddot{y}=Y(x, y),(x, y) \in \Omega=\Omega^{\circ} \subset \mathbb{R}^{2}, 0 \in \Omega
$$

where $X$ and $Y$ are at least $\mathcal{C}^{1}$. 
If $A$ defined by (3.6) with (3.10) is positive definite, i. e. $\Delta>0$ in (3.16) then we have the following

Proposition 15. Under the condition of proposition (11), if $\pi$ has a strict minimum at the origin, then the system (4.1) is Liapunov stable. (The Dirichlet-Lagrange theorem with respect to the new metric.)

If the matrix (3.6) is not positive definite, that is, if $\alpha$ in (3.6) is positive in a neighborhood of the origin, but the determinant $\Delta<0$, one may ask: "What happen with the stability of the origin for the system (4.1)?" First of all, a local Morse minimum of $\mathcal{C}^{2}$ functions is a non-degenerated extremum (the Jacobian of the function vanish) and the Hessian of the function is positive. Toward to understand this question we state the following result

Proposition 16. Let $(0,0)$ an isolated equilibrium for (4.1). Suppose that exist an indefinite quadratic first integral for this system, that is, (3.17) is negative with $\alpha$ in (3.10) positive in a neighborhood $\Omega \supset(0,0)$. Then, if $\pi$ has a non-degenerated local minimum (a Morse minimum) at $(0,0)$, the origin is an unstable equilibrium of (4.1).

Proof. Since the integral in (3.18) exist for the system (4.1) then (3.15) holds. If we linearized at $(0,0)$ we obtain the following system

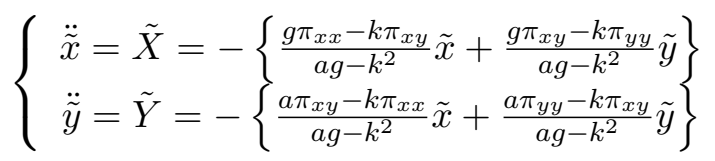

Now if we transform the two second order equation into four first order equation we have

$$
\dot{U}=B U,
$$

where $U=(v \tilde{X} w \tilde{Y})^{T}$ and

$$
B=\frac{1}{\left(a g-k^{2}\right)^{2}}\left(\begin{array}{cccc}
0 & 1 & 0 & 0 \\
-F_{1} & 0 & -F_{2} & 0 \\
0 & 0 & 0 & 1 \\
-F_{3} & 0 & -F_{4} & 0
\end{array}\right)
$$

with

$$
\begin{aligned}
& F_{1}=g \pi_{x x}-k \pi_{x y}, \\
& F_{2}=g \pi_{x y}-k \pi_{y y}, \\
& F_{3}=a \pi_{x y}-k \pi_{x x}, \\
& F_{4}=a \pi_{y y}-k \pi_{x y} .
\end{aligned}
$$

The eigenvalues is the roots of the following polynomial

$$
\lambda^{4}+\left(F_{1}+F_{4}\right) \lambda^{2}+\left(F_{1} F_{4}-F_{2} F_{3}\right),
$$

and the respective discriminant is

$$
\delta=\left(F_{1}+F_{4}\right)^{2}-4\left(F_{1} F_{4}-F_{2} F_{3}\right) .
$$

Since $F_{1} F_{4}-F_{2} F_{3}=\left(\pi_{x x} \pi_{y y}-\pi_{x y}^{2}\right)\left(a g-k^{2}\right)$, the discriminant is positive if $\pi_{x x} \pi_{y y}-\pi_{x y}^{2}>0$, since we have $\Delta<0$, i. e. $a g-k^{2}<0$. If we have $\pi$ with a Morse minimum at the origin then we have $\pi_{x x} \pi_{y y}-\pi_{x y}^{2}>0$. Thus the discriminant $\delta$ is positive.

To conclude, if we have the discriminant $\delta>0$, we have at least one eigenvalues positive which implies the instability. 
This theorem is equivalent to theorem of linearization for force fields which are attractors and have quadratic first integral. This turns to be very useful for a better insight of the geometry problem in mechanics.

\subsubsection{The Central Case $X(x, y)=-x f(x, y), Y(x, y)=-y f(x, y)$}

In this section we work with the system

$$
\begin{aligned}
& \ddot{x}=-x f(x, y) \\
& \ddot{y}=-y f(x, y),
\end{aligned}
$$

$f: \Omega=\Omega^{\circ} \subset \mathbb{R}^{2} \rightarrow \mathbb{R}, 0 \in \Omega, f \in \mathcal{C}^{2}, f(0,0)>0$. Remark that in the case above, we now have another first integral, this mean $S=x \dot{y}-y \dot{x}$. This integral is known as the Area Integral, and its occur for any central force field.

On this subject, Cesar and Barone-Netto [16] published some results when the kinetic part of the first integral (3.18) has constant coefficients. In this case, $f$ has a particular structure, namely there exists a function

$$
g(u): I=I^{\circ} \rightarrow \mathbb{R}, 0 \in I, g \in \mathcal{C}^{2},
$$

such that $f(x, y)=g(u)$, with $u=a x^{2}+k x y+g y^{2}$.

With (3.13) in this system we have

$$
\pi_{x}=(a x+k y) f, \quad \pi_{y}=(g x+k x) f .
$$

So, the condition $\pi_{x y}=\pi_{y x}$ gives

$$
(a x+k y) f_{y}=(g x+k x) f_{x},
$$

and the general solution of this equation is

$$
f(x, y)=g(u),
$$

with $u=a x^{2}+k x y+g y^{2}$, the function $g \in \mathcal{C}^{2}$ being arbitrary.

The system (4.4) then becomes

$$
\ddot{x}=-x g(u), \quad \ddot{y}=-y g(u), \quad u=a x^{2}+k x y+g y^{2} .
$$

The contribution by Cesar and Barone-Netto was that they proved

- The origin is stable for (4.4), if $u$ is (positive or negative) definite. Indeed, since $g(0)>0$ $(f(0,0)>0)$, then $Z$ has a strong extremum at the origin and $V$ (or $-V$ ) is a Liapunov function for stability.

- Whenever $u$ is semi-definite, up to a linear change of variables, we can write $u=\epsilon x^{2}(\epsilon= \pm 1)$ and the system (4.4) becomes

$$
\ddot{x}=-x f(x), \quad \ddot{y}=-y f(x),
$$

where $f$ is an even function.

\subsubsection{Not a Beautiful World (or maybe yes)}

In the first paragraph of this section we stated a new result about instability of some positional systems (see proposition (16)) which are attractors. Basically we applied the linearization about the origin and study the linear stability. Well, this technique is not always useful for this matter as we clued. In this part we will show an example that linearization doesn't work, which show once 
more that the problems of stability have not a general form to solve all of them. After this example we state another new result on instability of the origin for other types of systems.

Let us begin with semi-attractor definition and give a result for this type of field as a consequence of Chetaev's theorem. After we will define quasi-central force fields and give another results about those fields. All these results show example that we can't conclude anything if we use the proposition (16).

Definition. We call a Newton system (4.1) such that

$$
x X(x, y)+y Y(x, y)<0,
$$

a semi-attractor force field.

Proposition 17. Given a semi-attractor force field, such that the position vector $q=(x, y)$ and the force vector $F(q)=(X, Y)$ are linearly independent for all $(x, y) \in \Omega \backslash\{(0,0)\}$. Then the origin is an unstable equilibrium point for the system.

Proof. Consider $V(q, \dot{q})=x \dot{y}-y \dot{x}$, the hypothesis guarantee that

$$
\dot{V}=-x \ddot{y}+y \ddot{x}=-x Y+y X=\operatorname{det}[q \mid F(q)]
$$

does not change sign, so without lost of generality we consider $\dot{V}>0$, for $(x, y) \neq 0$.

Let $W=\left\{(q, \dot{q}) \in \mathbb{R}^{4}: V(q, \dot{q})>0\right\}$. It's clear that $0 \in \partial W$, and $\dot{V}>0$ in $W$, so we apply Chetaev's theorem directly which implies the thesis.

Example 4. Let the force field given by

$$
\left\{\begin{array}{l}
\ddot{x}=-x^{3}+y^{3} \\
\ddot{y}=-x^{3}-y^{3} .
\end{array}\right.
$$

Take as the neighborhood of the origin the following set $\mathcal{N}_{\epsilon}=\{(x, y) \in \mathcal{N}(0):\|(x, y)-(x, \lambda x)\|<\epsilon\}$ for a fixed $\lambda \in \mathbb{R}$, that is a neighborhood of the straight line $(x, \lambda x)$. Then the force is quasi-attractor in $\mathcal{N}_{\epsilon}$, since

$$
x \ddot{x}+y \ddot{y}=-x^{4}-y^{4}+x y^{3}-x^{3} y,
$$

and at the line $(x, \lambda x)$ we have

$$
-x^{4}-y^{4}+x y^{3}-x^{3} y=-x^{4}\left(\lambda^{4}-\lambda^{3}+\lambda+1\right)<0, \forall \lambda \in \mathbb{R} .
$$

And on the same line, the vectors $(x, y)$ and $(\ddot{x}, \ddot{y})$ form a positive base, since we have

$$
\left(-x^{3}+y^{3}\right) y-\left(-x^{3}-y^{3}\right) x=x^{4}\left(\lambda^{4}+\lambda^{3}-\lambda+1\right)>0, \forall \lambda \in \mathbb{R} .
$$

Then the result applies for this force field, thus the origin is an unstable equilibrium point.

The example above shows a case where the linearization does not work, since the matrix of linearization has determinant zero.

Definition. We call a Newton system

$$
\left\{\begin{array}{l}
\ddot{x}=-x f(x, y) \\
\ddot{y}=-y g(x, y)
\end{array}\right.
$$

with $f(x, y)>0$ and $g(x, y)>0$ for all $(x, y) \in \Omega$, a quasi-central force field.

As a consequence, a quasi-central force field is a semi-attractor force field by definition.

Let $p(x, y)=x y$ and $U=\left\{(x, y) \in \mathbb{R}^{2}:|p(x, y)|<1\right\}$. Let us define $f(x, y)=1-p(x, y)$ and $g(x, y)=1+p(x, y)$, trivially, $f$ and $g$ are strictly positive in $U$. 
Let us define the system

$$
\left\{\begin{array}{l}
\ddot{x}=-x(1-x y) \\
\ddot{y}=-y(1+x y)
\end{array}\right.
$$

which is an example of a quasi-central attractor.

If we apply the argument of linearization to this system, we obtain that the eigenvalues are all pure complex and they are on the unitary circle. But the system is not stable. Let us show this fact.

Let $V(x, \dot{x}, y, \dot{y})=y \dot{x}-x \dot{y}$ an auxiliary function with the following properties

1. $\dot{V}=y \ddot{x}-x \ddot{y}=2 x^{2} y^{2} \geq 0$;

2. $\dot{V}^{-1}(0)=W=\{(x, \dot{x}, y, \dot{y}): x=0\} \cup\{(x, \dot{x}, y, \dot{y}): y=0\}$;

3. If a solution of (4.6) is contained in $W$ then the solution is contained in $\Gamma=\{(x, \dot{x}, y, \dot{y}): x=\dot{x}=0\} \cup$ $\{(x, \dot{x}, y, \dot{y}): y=\dot{y}=0\}$. Thus, every invariant subset of $W$ stay in $\Gamma$ and $\Gamma \subset V^{-1}(0)$

Lemma 1. The origin is a Liapunov unstable equilibrium for (4.6)

Proof. Suppose the origin is Liapunov stable equilibrium for (4.6). Then, given a $\epsilon$, there is a $\delta>0$ such that $\left\|\left(x_{0}, \dot{x}_{0}, y_{0}, \dot{y}_{0}\right)\right\| \leq \delta$ thus any solution with initial condition $\left(x_{0}, \dot{x}_{0}, y_{0}, \dot{y}_{0}\right)$ stay in the closed ball $\mathcal{B}_{\epsilon}(0) \subset \mathbb{R}^{4}$ with center at the origin and radius $\epsilon$.

Consider a solution of (4.6), $\varphi(t)$ with initial conditions $y_{0}=\dot{x}_{0}=\frac{\delta}{2}$ and $x_{0}=\dot{y}_{0}=0$. One has $\|\varphi(t)\| \leq \epsilon$ since $\left\|\left(x_{0}, \dot{x}_{0}, y_{0}, \dot{y}_{0}\right)\right\|<\delta$, moreover $V\left(x_{0}, \dot{x}_{0}, y_{0}, \dot{y}_{0}\right)=\frac{\delta^{2}}{4}>0$, thus $V(\varphi(t)) \leq$ $\frac{\delta^{2}}{4}, \forall t \geq 0$, for (4.6).

Since $\mathcal{B}_{\epsilon}(0)$ is compact, the $\omega$-limit set of $\varphi$ is not null and it is invariant by the flows of (4.6). And within this $\omega$-limit, $V \geq \frac{\delta^{2}}{4}$.

In other hand, for (4.6), we have that this $\omega=\omega$-limit is contained in $W$. Thus by the third item above, $\omega \subset V^{-1}(0)$, which is a contradiction with the fact that for $z \in \omega$ we have $V(z) \geq \frac{\delta^{2}}{4}$.

Though we apply for an example, the result above is still true for more general quasi-central forces. Let the force field

$$
\begin{aligned}
& \ddot{x}=-x f(x, y), \\
& \ddot{y}=-y g(x, y) .
\end{aligned}
$$

And the following general hypothesis: $x y(g(x, y)-f(x, y)) \geq 0$ and the equality is true if, and only if $x y=0$.

Theorem 8. Let a quasi-central force field (4.8) with the general hypothesis above. Then the origin is Liapunov unstable equilibrium for the system.

Proof. The proof is identical to lemma (1) above.

The theorem (8) is an illustrative fact about the geometry of quasi-attractors forces, that is, if the force field is quasi-attractor and the vectors position $(x, y)$ and the force vector $(\ddot{x}, \ddot{y})$ are linearly independent but on the axis (i. e. $x y \neq 0$ ), and without lost of generality we suppose the base generated by the two vector is positive, then we always have a result like the theorem, that means the force field has an unstable equilibrium point at the origin. Use the fact that the base is positive and apply in the total derivative of $V(q, \dot{q})$.

As a consequence of the theorem above, we have

Corollary 7. Let a perturbative quasi-central attractor force

$$
\left\{\begin{array}{l}
\ddot{x}=-x\left(f(x, y)+h_{1}(x, y)\right) \\
\ddot{y}=-y\left(f(x, y)+h_{2}(x, y)\right)
\end{array}\right.
$$

where $|f(x, y)|>>\left|h_{i}(x, y)\right| \forall(x, y) \in \Omega$ and $h_{2}(x, y)-h_{1}(x, y) \geq 0,(x, y) \in \Omega$ (or $\left.\leq 0\right)$ and the equality is true if, and only if $x y=0$. Then the origin is a Liapunov unstable equilibrium. 


\subsection{Systems With Form $\mathrm{X}(\mathrm{x}, \mathrm{y})=-\mathrm{xf}(\mathrm{x}), \mathrm{Y}(\mathrm{x}, \mathrm{y})=-\mathrm{yf}(\mathrm{x})$}

In this section, we consider the system

$$
\left\{\begin{array}{l}
\ddot{x}=-x f(x) \\
\ddot{y}=-y f(x), \quad(x, y) \in \Omega=\Omega^{\circ} \subset \mathbb{R}^{2}, 0 \in \Omega,
\end{array}\right.
$$

where $f$ is continuous and guarantees uniqueness.

If $f(0) \leq 0$, then the equilibrium is unstable, for this, it is sufficient to consider the solutions of the system (4.10) with $x(0)=\dot{x}(0)=0$, i. e. the motions along the $y$-axis. So we assume that $f(0)>0$.

Its already known that the system (4.10) has two first integral, one is related from the fact that the system is central, the area integral, and the other came from the fact that the first part of the system is a mechanical system independent from the second one, "energy",

$$
\begin{gathered}
\dot{y} x-\dot{x} y=c, \\
\dot{x}^{2}+2 V(x)=2 E, \quad V(x)=\int_{0}^{x} \eta f(\eta) d \eta .
\end{gathered}
$$

Indeed, the energy can be obtained from a general theory which was exposed early.

One consequence which we have from energy is $V^{\prime \prime}(0)>0$ since $f(0)>0$ by hypothesis, which shows that the origin is a stable isolated equilibrium for the first part of system (4.10). Thus all orbits are periodic in a neighborhood of $(0,0)$ in the $x \dot{x}$-plane and have the origin in their interior, or the equilibrium point is a center.

Consider one of these periodic orbits and let $t \longmapsto x\left(t ; x_{0}, \dot{x}_{0}\right), x(t)$ for simplicity, be the corresponding motion with $x\left(0 ; x_{0}, \dot{x}_{0}\right)=x_{0}, \dot{x}\left(0 ; x_{0}, \dot{x}_{0}\right)=\dot{x}_{0}$ and period $T\left(x_{0}, \dot{x}_{0}\right)=T$. We consider the case $x_{0}>0$ and $\dot{x}_{0}=0$, the general situation can be obtained from this case by a translation in time.

Now plug this periodic orbit $x(t)$ into the second part of (4.10)

$$
\ddot{y}=-y f\left(x\left(t, x_{0}, 0\right)\right) \text {, where } x\left(t+T, x_{0}, 0\right)=x\left(t, x_{0}, 0\right),
$$

then we have a Hill's Equation and the study of this system is based on Floquet's Theory. Thus, we have two independent normalized solutions $y_{1}$ and $y_{2}$ with conditions (2.18).

Remark: We can define $y_{1}: t \longmapsto x\left(t ; x_{0}, 0\right) / x_{0}$ as the even solution of $(4.13)$, since $x\left(t ; x_{0}, 0\right)=$ $x\left(-t ; x_{0}, 0\right)$. Then we have

$$
y_{1}(0)=1, \quad \dot{y}_{1}(0)=0, \quad y_{1}(t+T)=y_{1}(t), \quad y_{1}(-t)=y_{1}(t),
$$

where the last equality immediately comes from (4.10) ${ }_{1}$. Moreover the integral (4.12) implies

$$
E=V\left(x_{0}\right), y_{1}\left( \pm t_{1}\right)=0, \dot{y}_{1}\left( \pm t_{1}\right)=\mp \sqrt{2 V\left(x_{0}\right)}, y_{1}(T / 2)=h\left(x_{0}\right) / x_{0}, \dot{y}_{1}(T / 2)=0,
$$

where $t_{1}=\min \left\{t \in \mathbb{R}_{+} ; y_{1}=0\right\}$ and $h\left(x_{0}\right)=\max \left\{x \in \mathbb{R}_{-} ; V(x)=V\left(x_{0}\right)\right\}$.

In order to obtain the general solution of the equation (4.13) let us consider the odd solution $y_{2}(t)$. So (4.11) with $c=1$ and (4.15) give

$$
y_{2}\left( \pm t_{1}+n T\right)= \pm\left\{2 V\left(x_{0}\right)\right\}^{-1 / 2}, \dot{y}_{2}(T / 2+n T)=x_{0} / h\left(x_{0}\right), \dot{y}_{2}(n T)=1,
$$

for any $n \in \mathbb{Z}$.

If $t \longmapsto \varphi(t)$ is a solution of the equation (4.13), then

$$
\varphi(t)=\varphi(0) y_{1}(t)+\dot{\varphi}(0) y_{2}(t) .
$$


But the function $t \longmapsto y_{2}(t+T)$ is a solution, therefore

$$
y_{2}(t+T)=y_{2}(T) y_{1}(t)+y_{2}(t),
$$

where we have used $\dot{y}_{2}(n T)=1$. Then for $t=n T$ we have

$$
\begin{gathered}
y_{2}((n+1) T)-y_{2}(n T)=y_{2}(T) \\
\Rightarrow y_{2}(n T)=n y_{2}(T), \text { for any } n \in \mathbb{Z} .
\end{gathered}
$$

Thus we have the following result

$$
\varphi(n T)=\varphi(0)+n \dot{\varphi}(0) y_{2}(T), \text { for any } n \in \mathbb{Z} .
$$

If we evaluate $y_{2}(t+T)$ at $t=-T / 2$ we see that $y_{2}(T)=0$ if and only if $y_{2}(T / 2)=0$, condition we already obtained from Floquet's Theory, and we call as Condition A. And we have

Proposition 18. Either all the solutions of the equation (4.13) are T-periodic, or any solution $t \longmapsto \varphi(t)$ with $\dot{\varphi}(0) \neq 0$ is global and unbounded.

The former case happens if and only if $y_{2}(T)=0$ or, equivalently, $y_{2}(T / 2)=0$.

Remark 6. A remark can be made here, the proposition (18) says that the system (4.10) is stable if and only it is totally periodic, with all solutions T-periodic. This is not true in general, for instance in dimension 3, it is open, the question: "If all orbits in the neighborhood of origin is T-periodic then the origin is stable?". Even if we substitute $f(x)$ by another function $g(x)$ with same properties of $f$ in $(4.10)_{2}$ if that assertion is true.

For instance if $\dot{\varphi}(0)=0$, then $\varphi(t)=\varphi(0) y_{1}(t)$. Thus the trajectory of $t \longmapsto\left(x\left(t, x_{0}, 0\right), \varphi(t)\right)$ is a straight segment passing through the origin.

If $\dot{\varphi}(0) y_{2}(t) \neq 0$, then the trajectory in the configuration plane can be like the configuration space in figure (4.1).

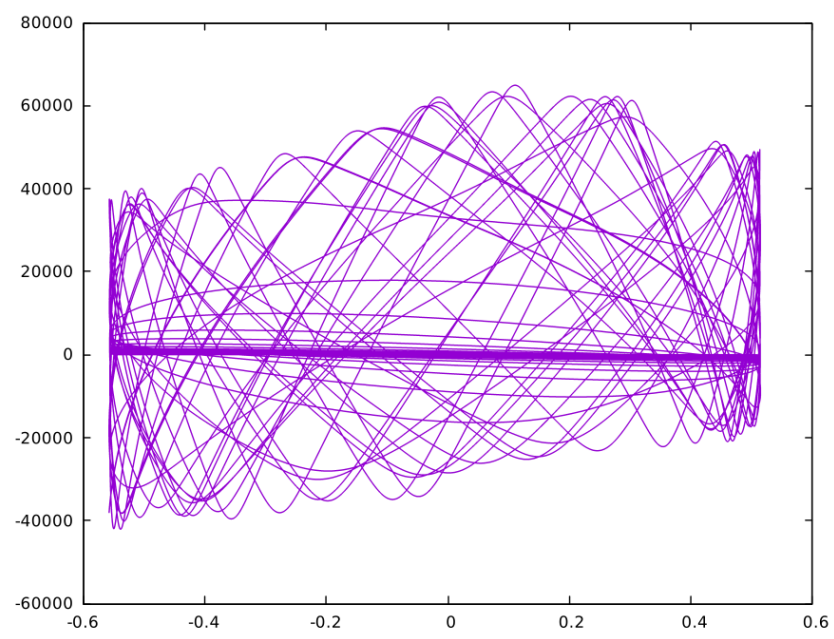

Figure 4.1: Configuration space for system (4.10) with $f(x)=0.5+x^{2}+x^{3}$.

In [38] Zampieri \& Barone-Netto, obtained a condition equivalent to condition A, in the following way. Consider the solution $\eta_{x_{0}}(t)$ of (4.13) with $\eta_{x_{0}}\left(T_{x_{0}} / 2\right)=0$ and $\dot{\eta}_{x_{0}}\left(T_{x_{0}} / 2\right)=\dot{y}_{2}\left(T_{x_{0}} / 2\right)$, where $T_{x_{0}}$ is the period of the orbit $x\left(t, x_{0}, 0\right)$ of the first equation in (4.10). The Area Integral has the same values for $\left(x\left(t, x_{0}, 0\right), \eta_{x_{0}}\right)$ and $\left(x\left(t, x_{0}, 0\right), y_{2}(t)\right)$. Therefore the two trajectories pass through the same point of the $y$ axis (in the configuration plane) at the instant $t_{x_{0}}$. If at that instant the two 
velocities also coincide, then the motions coincide and, in particular $y_{2}\left(T_{x_{0}} / 2\right)=\eta_{x_{0}}\left(T_{x_{0}} / 2\right)=0$. Thus

Proposition 19. All the solutions of the equation (4.13) are $T_{x_{0}}$-periodic if and only if

$$
\dot{y}_{2}\left(t_{x_{0}}\right)=\dot{\eta}_{x_{0}}\left(t_{x_{0}}\right) \text {. }
$$

The usefulness of this equivalent condition is related to the formula

$$
\frac{\dot{y}_{2}\left(t_{x_{0}}\right)}{x_{0}}=\lim _{x \rightarrow 0^{+}}\left\{\frac{1}{x}-\left[2 V\left(x_{0}\right)\right]^{1 / 2} \int_{x}^{x_{0}} \xi^{-2}\left[2\left(V\left(x_{0}\right)-V(\xi)\right)\right]^{-1 / 2} d \xi\right\}
$$

and for analogous expression for $\dot{\eta}_{x_{0}}\left(t_{x_{0}}\right) / x_{0}$ which can be obtained from the right hand side of (4.19) by using $0^{-}$and $h\left(x_{0}\right)$ instead of $0^{+}$and $x_{0}$. And we have

Theorem 9. The origin is a stable equilibrium for the system (4.10) if and only if there exists $a \in \mathbb{R}_{+}^{*}$ such that

$$
\dot{y}_{2}\left(t_{x_{0}}\right)=\dot{\eta}_{x_{0}}\left(t_{x_{0}}\right)
$$

holds for any $x_{0} \in(0, a)$.

Find an integral like (3.18) for (4.10) we obtain

$$
(x \dot{y}-y \dot{x})^{2} .
$$

This integral we can obtain directly since the system (4.10) is a central force field, thus the area integral and its multiple is an integral of the system. Another integral we obtain is

$$
\frac{1}{2} \dot{x}^{2}+\int_{0}^{x} \eta f(\eta) d \eta
$$

since the first equation of the system is independent from second. This is the energy-like integral.

These have the required form in (3.18). Now, we look for conditions on $f$, which the system (4.10) admits some first integral of the form (3.18) and independent of those we already known. The condition in (3.14) yields

$$
\left[\frac{3}{2} e f(x)+\left(d+\frac{1}{2} e x\right) f^{\prime}(x)\right] y+\left[-\frac{3}{2} b f(x)+\left(k-\frac{1}{2} b x\right) f^{\prime}(x)\right] x=0 .
$$

If we want that each coefficient vanish then we arrive

$$
-\frac{3}{2} b f(x)+\left(k-\frac{1}{2} b x\right) f^{\prime}(x)=0,
$$

which gives

$$
f(x)=\frac{c_{1}^{3}}{(2 k-b x)^{3}}
$$

And the first term

$$
\frac{3}{2} e f(x)+\left(d+\frac{1}{2} e x\right) f^{\prime}(x)=0
$$

which gives

$$
f(x)=\frac{c_{2}^{3}}{(2 d+e x)^{3}} .
$$

The expressions of $f(x)$ imply that $0 \neq p=2 k / c_{1}=2 d / c_{2}$ and $q=-b / c_{1}=e / c_{2}$. Thus

$$
b p+2 q k=0, e p-2 d q=0, f(x)=\frac{1}{(p+q x)^{3}} .
$$


For instance, $(d=1, e=2 q / p, a=b=c=k=0)$, we have the following first integral

$$
-\frac{q}{p} y \dot{x} \dot{y}+\left(\frac{1}{2}+\frac{q}{p} x\right) \dot{y}^{2}+\frac{y^{2}}{2 p(p+q x)^{2}} .
$$

And for $(a=1, b=c=d=e=k=0)$

$$
\frac{1}{2} \dot{x}^{2}+\frac{x^{2}}{2 p(p+q x)^{2}} \text {. }
$$

Thus we arrive

Proposition 20. The origin is stable for the system (4.10), with $f$ as in (4.20), for any positive $p$. Proof. Add the two independent maps above and use proposition (15).

Which give us a result already known, Darboux [14] and Halphen [17]

Proposition 21. Any trajectory of the system (4.10), with $f$ as in (4.20), is a conic.

\subsubsection{Liapunov Stability of Origin for System (4.10)}

Let the maps $f$ and $V$ be as in (4.10) and (4.12), with $f(0)=1$, without lost of generality, and let us consider

$$
\bar{u}: x \mapsto(\operatorname{sgn} x)[2 V(x)]^{1 / 2} \text {, thus } \bar{u}^{2}=2 V(x),
$$

where $\operatorname{sgn} x$ is the sign of $x$. Clearly

$$
\lim _{x \rightarrow 0}\left[2 V(x) / x^{2}\right]=f(0)=1, \text { which implies } \bar{u}^{\prime}(0)=1 .
$$

Furthermore, $\bar{u}^{\prime}(x)=(x f(x) / \bar{u}(x)) \rightarrow \bar{u}^{\prime}(0)>0$ as $x \rightarrow 0$. Therefore there exists a maximal open interval $J$, with $0 \in J$, such that $u=\bar{u} \mid J$ is a $\mathcal{C}^{1}$ diffeomorphism onto a symmetric interval $I$. We call $u$ the auxiliary potential for system (4.10).

Let us define

$$
h: J \rightarrow J, \quad x \mapsto u^{-1}(-u(x)), \text { thus } V(h(x))=V(x)
$$

This definition agrees with our definition of $h(x)$ in subsection (4.1.1) and we have

$$
h \in \operatorname{Diff}^{1}(J), h \circ h=i d, h(0)=0, h^{\prime}(0)=-1 .
$$

Remark 7. In fact this function $h(x)$ is know as Involution $(\neq I d)$. It is a solution of Babbage's Functional Equation with period two [7].

Remark 8. A counterclockwise $\pi / 4$-rotation of the graph of $h$ yields the graph of an even map $\tilde{h} \in \mathcal{C}^{1}$ with $\tilde{h}(0)=0$.

Now let us introduce the map,

$$
k: J \backslash\{0\} \rightarrow \mathbb{R}, \quad x \mapsto \frac{2}{u(x)}+\frac{1}{h(x)}-\frac{1}{x} .
$$

In general the limit of $k(x)$ as $x \rightarrow 0$ does not exist. However, we have

$$
\begin{gathered}
k \in \mathcal{C}^{1}(J \backslash\{0\} ; \mathbb{R}), k(h(x))=-k(x), \text { for any } x \in J \backslash\{0\}, \\
\lim _{x \rightarrow 0} x h(x)=0, \quad \lim _{x \rightarrow 0} x^{2} k^{\prime}(x)=0 .
\end{gathered}
$$

In the case where $\left.f^{\prime \prime}\right|_{x=0}$ exist, so that $f^{\prime}$ exist in a neighbourhood of zero, a calculation leads to the following 
Lemma 2. If $f^{\prime}(0)$ exists, then

$$
\lim _{x \rightarrow 0} k(x)=0 .
$$

If $f^{\prime \prime}(0)$ exists, then we also have

$$
\lim _{x \rightarrow 0} k^{\prime}(x)=-\frac{f^{\prime \prime}(0)}{4}+\frac{\left(f^{\prime}(0)\right)^{2}}{3} .
$$

And define the map

$$
l: J \cap \mathbb{R}_{+}^{*} \rightarrow \mathbb{R}, \quad x \mapsto \frac{\dot{\eta}_{x}\left(t_{x}\right)-\dot{y}_{2}\left(t_{x}\right)}{x}
$$

Remark 9. $l\left(x_{0}\right)=0$ iff all the solutions of the equation (4.13) are periodic. Furthermore, the origin is a stable equilibrium for the system (4.10) iff there exists a $\in \mathbb{R}_{+}^{*}$ such that $l(x)=0$ for any positive $x<a$.

We arrive the following result by Zampieri [37],

Proposition 22. If $\left.k\right|_{(0, a)}=0$ for some $a \in \mathbb{R}_{+}^{*}$, then $\left.l\right|_{(0, a)}=0$

Let $[0, a) \subset J$ then the condition $k(x)=0$, for all $x \in(0, a) \cup h((0, a))$, is equivalent to $\left.k\right|_{(0, a)}=0$. Furthermore this hold iff

$$
v(x)=2\left[\frac{x h(x)}{h(x)-x}\right]^{2}
$$

or, equivalently,

$$
f(x)=\frac{4 h(x)}{(h(x)-x)^{3}}\left(x^{2} h^{\prime}(x)-h^{2}(x)\right)
$$

for all $x \in(0, a) \cup h((0, a))$.

Proposition 23. Let $\bar{f} \in \mathcal{C}^{0}(\boldsymbol{B} ; \mathbb{R}), \boldsymbol{B} \subset \mathbb{R}$, open with $0 \in \boldsymbol{B}, \bar{f}(0)=1$ and let $\bar{v}, \bar{u}, \bar{h}, \bar{k}, \bar{l}$ associated with $\bar{f}$ as $v, u, h, k, l$ are associated with $f$. If $\bar{h}$ and $h$ are defined and coincide over some interval $(0, a)$, and $l(x)=\bar{l}(x)$ for all $x \in(0, a)$, then $f(x)=\bar{f}(x)$ for all $x \in(0, a) \cup h([0, a))$.

Theorem 10. $\left.k\right|_{(0, a)}=0$ for some $a \in \mathbb{R}_{+}^{*}$ if and only if $\left.l\right|_{(0, a)}=0$.

Corollary 8. The origin is a stable equilibrium for (4.10), with $f \in \mathcal{C}^{0}$ and $f(0)=1$, if and only if (4.27), or equivalently (4.28), holds in some neighbourhood of the origin (origin excluded).

\subsubsection{Some Remarks When $f(x)$ is Analytic in System (4.10)}

Let us suppose that $f(x) \in \mathcal{C}^{\omega}$ then we have $V(x) \in \mathcal{C}^{\omega}$ and thus $u(x) \in \mathcal{C}^{\omega}$. Then $u(x)$ has the following form in the neighborhood of origin,

$$
u(x)=\sum_{n=1}^{\infty} c_{n} x^{n}, \quad c_{1}=1^{*},
$$

where the $*$ holds since we have

Lemma 3. Let $u(x)$ defined as in (4.21) then $u^{\prime}(0)=1$

Proof.

$$
\lim _{x \rightarrow 0} \frac{u^{2}(x)}{x^{2}}=\lim _{x \rightarrow 0} \frac{u(x) u^{\prime}(x)}{x}=\lim _{x \rightarrow 0} u^{\prime}(x)^{2}+u(x) u^{\prime \prime}(x)=\lim _{x \rightarrow 0} u^{\prime}(x)^{2},
$$

and we have

$$
\lim _{x \rightarrow 0} \frac{u^{2}(x)}{x^{2}}=\lim _{x \rightarrow 0} \frac{2 V(x)}{x^{2}}=\lim _{x \rightarrow 0} \frac{V^{\prime}(x)}{x}=\lim _{x \rightarrow 0} V^{\prime \prime}(x)=f(0)=1 .
$$


Then we have the result.

Thus, we can apply the Lagrange inversion theorem on $u(x)$ and write the inverse as

$$
u^{-1}(x)=\sum_{n=1}^{\infty} d_{n} x^{n}, \quad d_{1}=1,
$$

with

$$
c_{n}=F_{n}\left(d_{1}, \ldots, d_{n}\right)
$$

and

$$
d_{n}=G_{n}\left(c_{1}, \ldots, c_{n}\right)
$$

Let us now find condition for $k$ in $(4.24)$ which $\left.k\right|_{(0, a)}=0$ for some $a \in \mathbb{R}_{+}^{*}$. Let

$$
k(x)=\frac{2 x h(x)+x u(x)-h(x) u(x)}{x u(x) h(x)},
$$

then $\left.k\right|_{(0, a)}=0$ if and only if $2 x h(x)+x u(x)-h(x) u(x)=\left.\theta(x)\right|_{(0, a)}=0$, since $x, h(x)$ and $u(x)$ vanish only at the origin.

Let us use (4.22) in $\theta(x)$ and with a change of variables $s=u(x)$ we arrive

$$
\left.\left.\theta(x)\right|_{(0, a)} \equiv 0 \Leftrightarrow \Theta(s)\right|_{(0, b)}=2 u^{-1}(s) u^{-1}(-s)+s\left[u^{-1}(s)-u^{-1}(-s)\right] \equiv 0
$$

By (4.30) we have

$$
u^{-1}(s) u^{-1}(-s)=\sum_{n=1}^{\infty} e_{n} s^{2 n},
$$

where $e_{n}=\sum_{k=1}^{n-1}(-1)^{2} 2 d_{k} d_{2 n-k}+(-1)^{n} d_{n}^{2}$. And

$$
s\left[u^{-1}(s)-u^{-1}(-s)\right]=\sum_{n=1}^{\infty} 2 d_{2 n-1} s^{2 n} .
$$

The condition above is the same condition for $\left.\theta(x)\right|_{(h(a), 0)} \equiv 0$. We only need show that $\theta(h(x))=$ $\theta(x)$ for $x \in(h(a), 0) \cup(0, a)=I$. But

$$
\theta(h(x))=2 h(x)(h \circ h(x))+h(x) u(h(x))-(h \circ h(x)) u(h(x)),
$$

and use (4.22) we arrive

$$
\theta(h(x))=2 h(x) x-h(x) u(x)+x u(x)=\theta(x),
$$

then the two series $(\theta(x)$ and $\theta(h(x)))$ coincide in $I$. Thus we proved the following

Lemma 4. $\left.k\right|_{(h(a), 0) \cup(0, a)} \equiv 0$ if and only if $e_{n}+d_{2 n-1}=0, \forall n \in \mathbb{N}$ or, equivalently, $(-1)^{n} d_{n}^{2}+$ $2 \sum_{j=2}^{n-1}(-1)^{j} d_{j} d_{2 n-j}-d_{2 n-1} \equiv 0$, for $n \geq 2$, where $d_{n}$ are the coefficients of $u^{-1}(x)$ in (4.30) and $d_{1}=1$.

Proposition 24. If the origin is a Liapunov stable equilibrium for the system (4.10), with $f(0)=1$, $f \in \mathcal{C}^{\omega}$ and $u(x)$ given by (4.21) with $u(x)\left(u^{-1}(x)\right)$ as in (4.29) $((4.30))$, then

1. $d_{2 n+1}=D_{n}\left(d_{1}, d_{2}, d_{3}, \ldots, d_{2 n-1}, d_{2 n}\right)$;

2. Given an even function $w(x)$ with $w(0)=0$, which is the even part the real analytic function $u^{-1}(x)$. Then, there is one, and only one, odd function $\tilde{w}(x)$ with $\tilde{w}^{\prime}(0)=1$, such that $u^{-1}(x)=\tilde{w}(x)+w(x)$, i. e., given a sequence $\left(d_{2}, d_{4}, \ldots, d_{2 n}, \ldots\right)$ there is one, and only one, sequence $\left(d_{1}, d_{3}, \ldots, d_{2 n-1}, \ldots\right)$ such that $d_{1}=1$ and $u^{-1}(x)=\sum_{n=1}^{\infty} d_{2 n-1} x^{2 n-1}+$ $\sum_{n=1}^{\infty} d_{2 n} x^{2 n}$, which give us the Liapunov stability of the orgin. 
Proof. Since the origin is Liapunov stable for a given $f$ then, by theorem (10) and its corollary, we have $\left.k\right|_{(0, a)} \equiv 0$, so the equality $e_{n}+d_{2 n-1}=0$ holds $\forall n \in \mathbb{N}$, so equivalently $(-1)^{n} d_{n}^{2}+$ $2 \sum_{j=2}^{n-1}(-1)^{j} d_{j} d_{2 n-j}-d_{2 n-1} \equiv 0$, for $n \geq 2$. If we change $n=m+1$ we have the 1$)$ part.

The 2) part.

Existence.

Since $\omega(x)$ is the even part of $u^{-1}(x)$ then it can be write as $\omega(x)=\frac{u^{-1}(x)+u^{-1}(-x)}{2}$. So the natural choice of the odd part is $\tilde{\omega}(x)=\frac{u^{-1}(x)-u^{-1}(-x)}{2}$. Then we have

$$
\tilde{\omega}(x)+\omega(x)=u^{-1}(x),
$$

which is analytic by hypothesis.

Uniqueness.

Since the origin is Liapunov stable, by the theorem above (4) we have

$$
2 u^{-1}(x) u^{-1}(-x)+x\left[u^{-1}(x)-u^{-1}(-x)\right] \equiv 0,
$$

or

$$
u^{-1}(x) u^{-1}(-x)+x[\tilde{\omega}(x)] \equiv 0,
$$

then

$$
\tilde{\omega}(x) \equiv-\frac{u^{-1}(x) u^{-1}(-x)}{x} .
$$

Using the second item of (6) we arrive with

$$
\tilde{\omega}(x)=\sum_{n=1}^{\infty} e_{n} s^{2 n-1},
$$

where $e_{n}=\sum_{k=1}^{n-1}(-1)^{k} 2 d_{k} d_{2 n-k}+(-1)^{n} d_{n}^{2}$, and $d_{n}^{\prime}$ s are the coefficients of $u^{-1}(x)$.

Since $\tilde{\omega}(x)$ is the odds coefficients of $u^{-1}(x)$ then we have

$$
d_{2 n-1} \equiv \sum_{k=1}^{n-1}(-1)^{k} 2 d_{k} d_{2 n-k}+(-1)^{n} d_{n}^{2}
$$

The derivative $\tilde{\omega}^{\prime}(0)=d_{1}=1$ by $(4.30)$.

To construct an analytic function $f(x)$ in (4.10) we have to obtain the an analytic auxiliary potential $u(x)$ like $(4.21)$

Theorem 11. Given a sequence $\left(s_{1}, s_{2}, \ldots\right)$, there is one and only one sequence $\left(1, r_{2}, \ldots\right)$ such that

$$
u(x)=\sum_{n=1}^{\infty} s_{n} x^{2 n}+\sum_{n=1}^{\infty} r_{n} x^{2 n-1}
$$

is an analytic auxiliary potential of a stable system (4.10).

Proof. Let us suppose a given sequence $\left(s_{1}, s_{2}, \ldots\right)$, namely $\left(c_{2}, c_{4}, \ldots\right)$ and the assumptions that the system given construct be stable. Define $\left(1, r_{2}, \ldots\right)=\left(c_{1}, c_{3}, \ldots\right)$, then $d_{1}=1$ in (4.30), and

$$
d_{2}=G_{2}\left(c_{1}, c_{2}\right)
$$

. Using the stability hypothesis and the proposition before, we obtain $d_{3}=D_{1}\left(d_{1}, d_{2}\right)$. Thus, if we use the Lagrange inversion coefficients, we obtain

$$
c_{3}=F_{3}\left(d_{1}, d_{2}, d_{3}\right)
$$


With that, and the even part given we have $c_{1}, c_{2}, c_{3}, c_{4}$ and $d_{1}, d_{2}, d_{3}$. Now we repeat the process for the data we already have and we find

$$
d_{4}=G_{4}\left(c_{1}, c_{2}, c_{3}, c_{4}\right),
$$

and using stability and the proposition before we have $d_{5}=D_{2}\left(d_{1}, d_{2}, d_{3}, d_{4}\right)$. And then by Lagrange inversion coefficient we obtain $c_{5}$. By continue this algorithm, since we have the even part $\left(c_{2}, c_{4}, \ldots\right)$ we can obtain the odd part $\left(c_{1}, c_{3}, \ldots\right)$, an then we find

$$
d_{2 n}=G_{2 n}\left(c_{1}, c_{2}, \ldots, c_{2 n}\right) .
$$

Using the stability hypothesis we obtain

$$
d_{2 n+1}=D_{n}\left(d_{1}, d_{2}, \ldots, d_{2 n-1}, d_{2 n}\right)
$$

and then

$$
c_{2 n+1}=F_{2 n+1}\left(d_{1}, d_{2}, \ldots, d_{2 n}, d_{2 n+1}\right),
$$

and this complete our proof. So, from a sequence given we obtain the analytic auxiliary potential of a stable system.

The uniqueness is guaranteed by the construction of the coefficients of an auxiliary potential. 
STABILITY OF SOME POSITIONAL SYSTEMS 


\section{Bibliography}

[1] Alain Albouy. Projective dynamics and classical gravitation. Regular and Chaotic Dynamics, 13(6):525-542, 2008. 19

[2] Alain Albouy. Projective dynamics and first integrals. Regular and Chaotic Dynamics, 20(3):247-276, 2015. 19

[3] Paul Appell. De l'homographie en mécanique. American Journal of Mathematics, pages 103114, 1889. 3, 19, 24

[4] Paul Appell. Sur les lois de forces centrales faisant décrire à leur point d'application une conique quelles que soient les conditions initiales. American Journal of Mathematics, pages 153-158, 1891. 19

[5] Vladimir I Arnold, Valery V Kozlov, and Anatoly I Neishtadt. Mathematical aspects of classical and celestial mechanics, volume 3. Springer Science \& Business Media, 2007. 5, 19

[6] Vladimir Igorevich Arnol'd. Mathematical methods of classical mechanics, volume 60. Springer Science \& Business Media, 2013. 5, 29

[7] Charles Babbage. An essay towards the calculus of functions. Philosophical transactions of the Royal Society of London, 105:389-423, 1815. 37

[8] Angelo Barone-Netto and Mauro de Oliveira Cesar. Non-conservative positional systemsstability. Dynamics and Stability of Systems, 2(3-4):213-221, 1988. 4, 19, 29

[9] Angelo Barone-Netto, Mauro de Oliveira Cesar, and Gianluca Gorni. A computational method for the stability of a class of mechanical systems. Journal of Differential Equations, 184(1):1-19, 2002. 19, 29

[10] Marcel Berger. Geometry (vols. 1-2), 1987. 3, 5

[11] J Bertrand. Sur la possibilité de déduire dâune seule des lois de kepler le principe de lâattraction. Comtes rendues, 9:1877, 1877. 1, 19

[12] Joseph Bertrand. Mémoire sur les intégrales communes à plusieurs problèmes de mécanique. Journal de Mathématiques Pures et Appliquées, pages 121-174, 1852. 19

[13] Joseph Bertrand. Mémoire sur quelques-unes des formes les plus simples que puissent présenter les intégrales des équations différentielles du mouvement d'un point matériel. Journal de Mathématiques Pures et Appliquées, pages 113-140, 1857. 19

[14] G Darboux. Recherche de la loi que doit suivre une force centrale pour que la trajectoire qu'elle détermine soit toujours une conique. CR Acad. Sci. Paris, 16, 1877. 19, 37

[15] Mauro de Oliveira Cesar and Angelo Barone-Netto. The existence of liapunov functions for some non-conservative positional mechanical systems. Journal of differential equations, 91(2):235-244, 1991. 3, 19, 29 
[16] Mauro de Oliveira Cesar and Angelo Barone-Netto. Stability of some central forces. Nonlinear Differential Equations and Applications NoDEA, 6(3):289-296, 1999. 19, 31

[17] GH Halphen. Sur les lois de kepler. Bulletin de la Société Philomatique de Paris, pages 7-1, 1878. $1,19,37$

[18] Vladimir Andreevich Iakubovich and Viacheslev Mikhaĭlovich Starzhinskiı̌. Linear differential equations with periodic coefficients, volume 2. Wiley, 1975. 3,5

[19] Edward Kasner. The ithaca colloquium. Bulletin of the American Mathematical Society, $8(1): 22-25,1901.19$

[20] Edward Kasner. Physical curves. Proceedings of the National Academy of Sciences, 33(8):246251, 1947. 19

[21] Edward Kasner and John De Cicco. Generalization of appell's transformation. Studies in Applied Mathematics, 27(1-4):262-269, 1948. 19

[22] Edward Kasner and John DeCicco. A generalized theory of dynamical trajectories. Transactions of the American Mathematical Society, 54(1):23-38, 1943. 19

[23] Edward Kasner and Don Mittleman. A general theorem on the initial curvature of dynamical trajectories. Proceedings of the National Academy of Sciences, 28(2):48-52, 1942. 19

[24] VV Kozlov. Asymptotic motions and the inversion of the lagrange-dirichlet theorem. Journal of Applied Mathematics and Mechanics, 50(6):719-725, 1986. 29

[25] Steven G Krantz and Harold R Parks. A primer of real analytic functions. Springer Science \& Business Media, 2002. 3, 5

[26] Joseph-Louis Lagrange. Recherches sur le mouvement dâun corps qui est attiré vers deux centres fixes. Miscellanea Taurinensia, 4:1766-1769, 1766. 1, 3, 19

[27] Joseph Louis Lagrange. Sur le problème de Kepler. Chez Haude et Spener, Libraires de la Cour \& de l'Académie royale, 1771. 19

[28] Hans Lundmark. Newton systems of cofactor type in Euclidean and Riemannian spaces. Matematiska institutionen, Linköpings universitet, 2001. 4

[29] Aleksandr Mikhailovich Lyapunov. The general problem of the stability of motion. International Journal of Control, 55(3):531-534, 1992. 13, 29

[30] Wilhelm Magnus and Stanley Winkler. Hill's equation. Courier Corporation, 2013. 3, 5

[31] Pierre Simon marquis de Laplace. Traité de mécanique céleste, volume 5. Crapelet, 1825. 19

[32] Kenneth Meyer, Glen Hall, and Dan Offin. Introduction to Hamiltonian dynamical systems and the N-body problem, volume 90. Springer Science \& Business Media, 2008. 29

[33] Waldyr Muniz Oliva. Geometric mechanics. Springer, 2004. 5

[34] Stefan Rauch-Wojciechowski. Newton representation for stationary flows of the kdv hierarchy. Physics Letters A, 170(2):91-94, 1992. 2

[35] Stefan Rauch-Wojciechowski, Krzysztof Marciniak, and Hans Lundmark. Quasi-lagrangian systems of newton equations. Journal of Mathematical Physics, 40(12):6366-6398, 1999. 4

[36] Félix Tisserand. Traité de mécanique céleste. Gauthier-Villars, 1891. 19 
[37] Gaetano Zampieri. Liapunov stability for some central forces. Journal of differential equations, $74(2): 254-265,1988.3,4,19,29,38$

[38] Gaetano Zampieri and Angelo Barone-Netto. Attractive central forces may yield liapunov instability. Dynamical Systems and Partial Differential Equations, Editorial Equinoccio, Caracas, pages $105-112,1986.19,29,35$ 